San Jose State University

SJSU ScholarWorks

Faculty Publications, Biological Sciences

Biological Sciences

$1-1-2012$

\title{
Investigation of the importance of rock chemistry for saxicolous lichen communities of the New Idria serpentinite mass, San Benito County, California, USA
}

N Rajakaruna

San Jose State University, nrajakaruna@gmail.com

K Knudsen

University of California - Riverside

A Fryday

Michigan State University

RE. O’Del

United States Bureau of Land Management Hollister Field Office, 20 Hamilton Ct., Hollister, CA USA

N Pope

University of California - Davis

See next page for additional authors

Follow this and additional works at: https://scholarworks.sjsu.edu/biol_pub

Part of the Biology Commons, and the Plant Biology Commons

\section{Recommended Citation}

N Rajakaruna, K Knudsen, A Fryday, R E. O'Del, N Pope, F C. Olday, and S Woolhouse. "Investigation of the importance of rock chemistry for saxicolous lichen communities of the New Idria serpentinite mass, San Benito County, California, USA" The Lichenologist (2012): 695-714. https://doi.org/10.1017/

S0024282912000205

This Article is brought to you for free and open access by the Biological Sciences at SJSU ScholarWorks. It has been accepted for inclusion in Faculty Publications, Biological Sciences by an authorized administrator of SJSU ScholarWorks. For more information, please contact scholarworks@sjsu.edu. 


\section{Authors}

N Rajakaruna, K Knudsen, A Fryday, R E. O'Del, N Pope, F C. Olday, and S Woolhouse 


\section{The Lichenologist}

http://journals.cambridge.org/LIC

Additional services for The Lichenologist:

Email alerts: $\underline{\text { Click here }}$

Subscriptions: Click here

Commercial reprints: Click here

Terms of use : $\underline{\text { Click here }}$

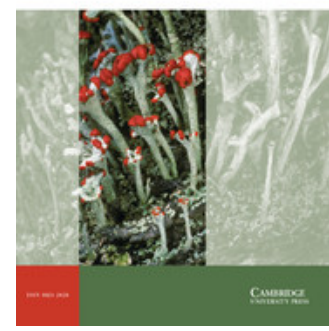

\section{Investigation of the importance of rock chemistry for saxicolous lichen communities of the New Idria serpentinite mass, San Benito County, California, USA}

Nishanta RAJAKARUNA, Kerry KNUDSEN, Alan M. FRYDAY, Ryan E. O'DELL, Nathaniel POPE, Fred C. OLDAY and Suzie WOOLHOUSE

The Lichenologist / Volume 44 / Issue 05 / September 2012, pp 695 - 714

DOI: 10.1017/S0024282912000205, Published online: 24 August 2012

Link to this article: http://journals.cambridge.org/abstract S0024282912000205

How to cite this article:

Nishanta RAJAKARUNA, Kerry KNUDSEN, Alan M. FRYDAY, Ryan E. O'DELL, Nathaniel POPE, Fred C. OLDAY and Suzie WOOLHOUSE (2012). Investigation of the importance of rock chemistry for saxicolous lichen communities of the New Idria serpentinite mass, San Benito County, California, USA. The Lichenologist,44, pp 695-714 doi:10.1017/S0024282912000205

Request Permissions : $\underline{\text { Click here }}$ 


\title{
Investigation of the importance of rock chemistry for saxicolous lichen communities of the New Idria serpentinite mass, San Benito County, California, USA
}

\author{
Nishanta RAJAKARUNA, Kerry KNUDSEN, Alan M. FRYDAY, Ryan E. O'DELL, \\ Nathaniel POPE, Fred C. OLDAY and Suzie WOOLHOUSE
}

\begin{abstract}
Although several lichen inventories exist for European ultramafic sites, only four surveys of serpentine lichens for North America have been published to date. Of those, only one has been conducted in California. We conducted a survey of saxicolous lichens from ultramafic rocks (including nephrite, partially serpentinized peridotite, and serpentinite) and non-ultramafic rocks (including silica-carbonate, shale, and sandstone) at the New Idria serpentinite mass, San Benito County, California. X-ray Fluorescence Analysis of the rocks from which the lichens were collected revealed significant elemental differences between the ultramafic and non-ultramafic rocks for 26 of the 32 major and trace elements analyzed. We identified a total of 119 species of lichenized and lichenicolous fungi; 60 species were restricted to ultramafic substrata, 19 to silica-carbonate, and 15 to shale and sandstone. Only 4 species were shared in common. A permutational multivariate analysis of variance (perMANOVA) test revealed significant differences in lichen assemblages between ultramafic and non-ultramafic rocks at the species level but not at the generic level, with species richness (alpha-diversity) significantly greater at the ultramafic sites. We suggest that, although differences in geochemistry clearly influence the lichen community composition, other factors, especially substratum age and the physical characteristics of the rock, are of equal, if not greater, importance. Of all the species collected, six, Buellia aethalea, B. ocellata, Caloplaca oblongula, Rhizocarpon saurinum, Thelocarpon laureri, and Trapelia obtegens, are reported new to California, along with an apparently previously undescribed Solenopsora $\mathrm{sp}$. The rest of the species encountered are relatively frequent in the lichen flora of southern and central California, except Aspicilia praecrenata, a rare California endemic that we collected on both ultramafic and non-ultramafic rocks.
\end{abstract}

Key words: edaphic endemism, geobotany, serpentine, ultramafic

Accepted for publication 14 April 2012

\section{Introduction}

Lichens have an intimate and often inseparable relationship with their substratum, even leading to unique lichen-geoedaphic asso-

N. Rajakaruna and F. C. Olday: College of the Atlantic, 105 Eden Street, Bar Harbor, ME 04609, USA. Email: nrajakaruna@coa.edu

K. Knudsen: Department of Botany \& Plant Sciences, 2117 Bachelor Hall, University of California, Riverside, CA 92521, USA.

A. M. Fryday: Herbarium, Department of Plant Biology, Michigan State University, East Lansing, MI 48824, USA.

R. E. O'Dell: United States Bureau of Land Management Hollister Field Office, 20 Hamilton Ct., Hollister, CA 95023, USA.

N. Pope: Department of Entomology, University of California Davis, One Shields Avenue, CA 95616, USA. S. Woolhouse: Department of Biological Sciences, One Washington Square, San José State University, San José, CA 95192, USA. ciations (Brodo 1973; Garty \& Galun 1974; Wilson 1995). Although the geochemistry and mineralogy of rocks may play an important role in the occurrence of individual lichen species and assembly of lichen communities (Purvis \& Halls 1996), the exact nature of such relationships or the mechanisms of such influences have not been thoroughly investigated.

Lichens are a dominant component of the biodiversity of many heavy metal-enriched sites, including mine tailings (Purvis \& Halls 1996; Purvis \& Pawlik-Skowrońska 2008; Rajakaruna et al. 2011) and ultramafic ('serpentine') substrata (Favero-Longo et al. 2004; Harris et al. 2007; Paukov 2009), at times displaying distinct species associations (Rajakaruna et al. 2011, and references therein). Despite extensive research on the effect of ultramafic substrata on vascular plants, little 
research has been undertaken to describe lichen communities growing on ultramafic substrata (Favero-Longo et al. 2004; Rajakaruna et al. 2009). Ultramafic rock is primarily composed of ferromagnesian silicates [ $<45 \%$ silica $(\mathrm{Si}) ;>18 \%$ magnesium oxide (MgO); Brooks 1987; Coleman \& Jove 1992]. Common ultramafic rock types include peridotites (dunite, wehrlite, harzburgite, lherzolite) and the secondary alteration products formed by their hydration within the Earth's crust, including serpentinite (Coleman \& Jove 1992). Ultramafic rocks and soils derived from them are generally deficient in plantessential nutrients such as nitrogen $(\mathrm{N})$, phosphorus $(\mathrm{P})$, potassium $(\mathrm{K})$, sulphur $(\mathrm{S})$; have a calcium $(\mathrm{Ca})$ to magnesium $(\mathrm{Mg})$ molar ratio (Ca:Mg) of less than 1 ; and have elevated levels of heavy metals such as nickel (Ni) and chromium (Cr) (O'Dell \& Rajakaruna 2011, and references therein). Due to the intense selective pressure generated by such stressful edaphic conditions, ultramafic substrata promote speciation and the evolution of ultramafic endemism in phanerogams (Kruckeberg 1986; Rajakaruna 2004; Kay et al. 2011), contributing to unique floras with high rates of endemism and species with disjunct distributions (Harrison \& Rajakaruna 2011). Interestingly, species-level ultramafic endemism is not a common phenomenon among cryptogams, including lichens (Alexander et al. 2007; Rajakaruna et al. 2009) where species- and communitylevel patterns appear to be more strongly influenced by macro- and micro-climate and the physical properties of the rock than by its mineral composition (Rajakaruna et al. 2009). In a comprehensive review of lichens found on ultramafic substrata worldwide, Favero-Longo et al. (2004) found co-occurrence of species characteristic of Ca-rich and Si-rich rocks and occurrence of species characterized by disjunct distribution patterns as common features of lichen communities in ultramafic environments. No consistent trends were detected in other features that are typical of phanerogams on ultramafics, such as paucity of species and occurrence of particular ecotypes. Several lichens collected from ultramafic substrata in Europe have been described as new to science, although it is unclear if these are truly ultramafic endemics, or species which are rare and were collected only from ultramafic substrata (Favero-Longo et al. 2004, with references therein). Moreover, most of the species first reported as restricted to ultramafic substrata are poorly differentiated from related species and have been collected from other substrata (Wirth 1972; Hafellner 1991). Whereas several recent lichen inventories exist for European ultramafic sites (Kossowska 2001; FaveroLongo et al. 2004, 2005; von Brackel 2007; Favero-Longo \& Piervittori 2009), including the earliest known published study of the relationship between lichens and ultramafic substrata (Hegetschweiler \& Stizenberger 1887), there are only a handful of published surveys to date of ultramafic lichens for North America (Ryan 1988; Sirois et al. 1988; Sigal 1989; Harris et al. 2007). Sirois et al. (1988) listed a total of 202 lichen taxa on Mt. Albert, Gaspésian Provincial Park, Québec, Canada, of which 157 were reported from partially serpentinized peridotite and 81 were restricted to this rock type. Of the taxa reported from ultramafic substrata, seven were new to North America, three were new to Canada, and 18 were new to Québec. They concluded that the ecological influences of ultramafic substrata on the lichens were similar to those observed on the region's vascular plants (Rune 1954), where many taxa are largely restricted to ultramafic substrata. A study of marine and maritime lichens collected from partially serpentinized peridotite rocks from Fidalgo Island, Skagit County, Washington, USA, found 61 species, including 15 species new to the state and one (Verrucaria sandstedei B. de Lesd.) new to North America (Ryan 1988). Only one study of lichens on ultramafic substrata is known to have been conducted in California (Sigal 1989), despite the strong focus there to elucidate the relationship between vascular plant species and ultramafic substrata (Alexander et al. 2007). Sigal (1989) reported 76 lichens from five ultramafic sites in central California. These included a collection of Ramonia gylactiformis (Zahlbr.) Vězda from peridotite and serpentinite at Complexion Springs in Lake 


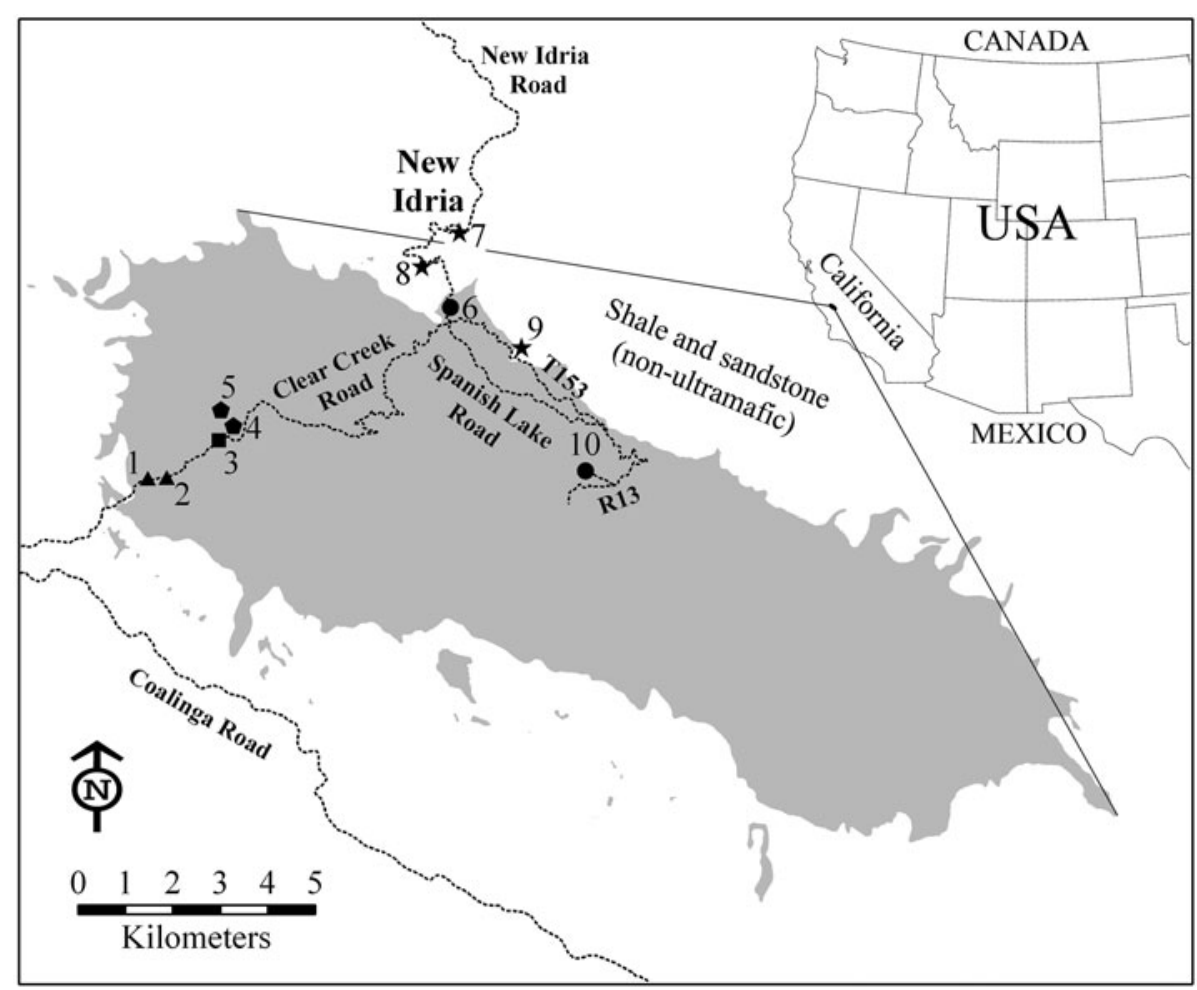

FIG. 1. Map of New Idria serpentinite mass showing sampling sites and their geological characteristics. Geology: $=$ New Idria serpentinite (ultramafic), $\square=$ Ghert, shale, and sandstone (non-ultramafic). Collection locality geology: $\boldsymbol{\Delta}=$ Nephrite (ultramafic), $\mathbf{a}=$ Serpentinized peridotite (ultramafic), $\boldsymbol{\theta}=$ Silica-carbonate (nonultramafic), $\star=$ Shale and sandstone (non-ultramafic), $=$ Serpentinite (ultramafic). Numbers refer to collection sites given in Table $1 . \mathrm{R} 13$ \& T153 are road numbers.

County that was recently recognized as a distinct species and described as new to science as Ramonia extensa Lendemer, K. Knudsen \& Coppins (Lendemer et al. 2009). The taxon is still known only from the type collection on ultramafic rock and deserves further study to determine if it is a strict ultramafic endemic. A recent study by Harris et al. (2007) explored the lichen flora of a partially serpentinized peridotite outcrop on Little Deer Isle, Hancock County, Maine. Sixtythree species in 35 genera were found, with two species, Buellia ocellata (Flörke) Körb. and Cladonia symphycarpia (Flörke) Fr., being new reports for New England. The handful of available studies suggest that there may be an ultramafic substratum effect for lichens in North America, and that further study may reveal new species or interesting floristic associations.

Our study examines the saxicolous lichen flora of the New Idria serpentinite mass, San Benito County, California, USA (Fig. 1). Whereas previous studies have explored the geology (Van Baalen 1995), soils (Alexander et al. 2007), and their relationship to plant species (Lazarus et al. 2011) of this area, no studies to date have surveyed the cryptogamic biota of the area, including lichens. We present the lichen biota of nephrite (jade), partially serpentinized peridotite, serpentinite, silica-carbonate, shale, and sandstone rocks associated with, or adjacent to, the New Idria serpentinite mass, with relevant geochemical information for the rocks from which the species were collected. 


\section{Materials and Methods}

\section{Site description and field methods}

The New Idria serpentinite mass, located in far southern San Benito and far western Fresno Counties, is one of the largest ultramafic masses in the South Coast Range of California, USA $\left(36 \cdot 3^{\circ} \mathrm{N}, 120 \cdot 6^{\circ} \mathrm{W}\right.$; Figs 1 , 2A). The lenticular mass of serpentinite is c. $22 \mathrm{~km}$ long, $8 \mathrm{~km}$ wide, and totals $13000 \mathrm{ha}$. It forms the centre of an asymmetrical anticlinal dome that is flanked by Jurassic and Cretaceous-aged sedimentary rocks (shale and sandstone) of the Franciscan and Panoche formations (Van Baalen 1995). The serpentinite mass was derived from peridotite (harzburgite and dunite), which has been completely minerologically altered, sheared, and crushed to yield a nearly incoherent mass of pulverized serpentinite, (Fig. 2B; Van Baalen 1995), although some small, scattered hard outcrops of nephrite, serpentinite and partially serpentinized peridotite remain (Fig. 3). The serpentinite of the outcrops is typically hard, but can flake off into large flakes and plates, and the surface texture varies from lamellar to granular to vacuolar porous. The partially serpentinized peridotite of the outcrops is typically hard, but can be crumbly, and generally has a coarse granular surface texture. Boulders of nephrite (very hard; granular surface texture) are distributed throughout the serpentinite mass. The New Idria serpentinite mass also contains massive inclusions of silica-carbonate rocks, many of which contain cinnabar (mercury ore) deposits (Fig. 4A). Silicacarbonate rocks are typically hard with a vacuolar porous surface texture and have a dominant mineral composition of quartz, chalcedony, opal, ankerite, magnesite, and dolomite (Van Baalen 1995). Silica-carbonate forms from the precipitation of minerals from hydrothermal fluids of ultramafic origin within the serpentinite mass (Van Baalen 1995). The rocks contain $\gg 45 \% \mathrm{Si}$ and $\ll 18 \% \mathrm{MgO}$ and, therefore, although they are derived from hydrothermal fluids of ultramafic origin, silicacarbonate is classified as a non-ultramafic rock in this study. Cinnabar deposits also occur in Panoche shale and sandstone on the north-eastern edge of the New Idria serpentinite mass at New Idria (New Idria Mine Tailings; New Idria Camp Pit 2) and San Carlos Peak (San Carlos Peak Mine Pit; Fig. 5B). Cinnabar was mined at New Idria and San Carlos Peak from 1851 to 1972 (Gilbert 1984) and numerous large open mine pits and cinnabar-bearing tailing piles (tips) still remain.

The New Idria serpentinite mass is subject to a Mediterranean-type climate (cool wet winters and hot dry summers) with mean annual precipitation of $40-60 \mathrm{~cm}$ (Alexander et al. 2007) that primarily occurs between October and April. Snow is occasional during winter (December-February) and short-lived. Elevation range across the sampling localities varies from $841 \mathrm{~m}$ to $1422 \mathrm{~m}$. Vegetative cover consists of chaparral at lower elevations and conifer forest at higher elevations. 'Moonscape' barrens, completely devoid of vegetation, are abundant and a prominent feature of the New Idria serpentinite mass (Figs 2, 3 \& 5A).
On 22 February 2010 and 21-22 April 2011, we collected lichens from ultramafic rocks including nephrite ( $n=\&$ sites), partially serpentinized peridotite $(n=4)$, and serpentinite $(n=2)$, and from non-ultramafic rocks including silica-carbonate $(n=2)$ and shale and sandstone $(n=3)$ adjacent to the New Idria serpentinite mass (Table 1; Fig. 1). For this study, sedimentary shale and sandstone are together considered a single rock type. All five non-ultramafic sites were extensively disturbed by mining as late as 1972, exposing fresh rock surfaces, in contrast to the little to no disturbance that has occurred at the ultramafic sites. As a result, the lichen community on the non-ultramafic sites represents a younger community than that on the ultramafic sites. Lichens were collected at each site until it was subjectively considered that the site had been well sampled. This varied from around 15 minutes (Sites 2, 3 and 8) to over an hour (Sites 1 and 10). It was considered that this was preferable to spending a fixed amount of time at each site, which would have resulted in disproportionate effort being expended on species-poor sites and would have resulted in these sites being over recorded. Representative rock samples, upon which the lichens were growing, were also collected. All lichen collections were identified by either the second or third authors, using standard reference works and comparison with named herbarium specimens or, for critical species, by experts in a particular group (see acknowledgments). All collections are permanently housed in the herbaria of either the College of the Atlantic (HCOA), University of California, Riverside (UCR), or Michigan State University (MSC). Nomenclature and naming authorities follow Index Fungorum Partnership (http://indexfungorum.org).

\section{Elemental analysis}

Elemental analysis (X-ray fluorescence) was conducted on pooled samples of 1-3 rock fragments from 1-2 different rock samples from each site where lichens were collected. Pooling of fragments and samples was necessary because of the high cost of the procedure. Elemental concentrations for each sample pooled were determined for major (Al-Ti) and trace (As-Zr) elements. The analyses were carried out by the GeoAnalytical Laboratory, Washington State University, WA, USA, using an automated Thermo ARL Advant'XP+ wavelength dispersive sequential unit running at $60 \mathrm{keV}$ and $60 \mathrm{~mA}$ with a rhodium target. Samples received as rock were prepared for analysis by chipping in a hardened steel jaw crusher then ground to a very fine powder in a tungsten carbide ring mill. The sample powder was weighed with di-lithium tetraborate flux at a 2:1 (low dilution) flux to rock ratio, mixed, then fused at $1000^{\circ} \mathrm{C}$ in a muffle oven for 45 min. Once cooled, the glass pellet was then re-ground, re-fused, and polished on diamond laps to provide a smooth flat surface for analysis. The concentration of elements was measured in $c .66 \mathrm{~min}$ under full vacuum with a $29 \mathrm{~mm}$ mask. The net intensities for all elements were corrected for line interferences and background slopes. Inter-element absorption and secondary enhancement effects were calculated using the fundamental 

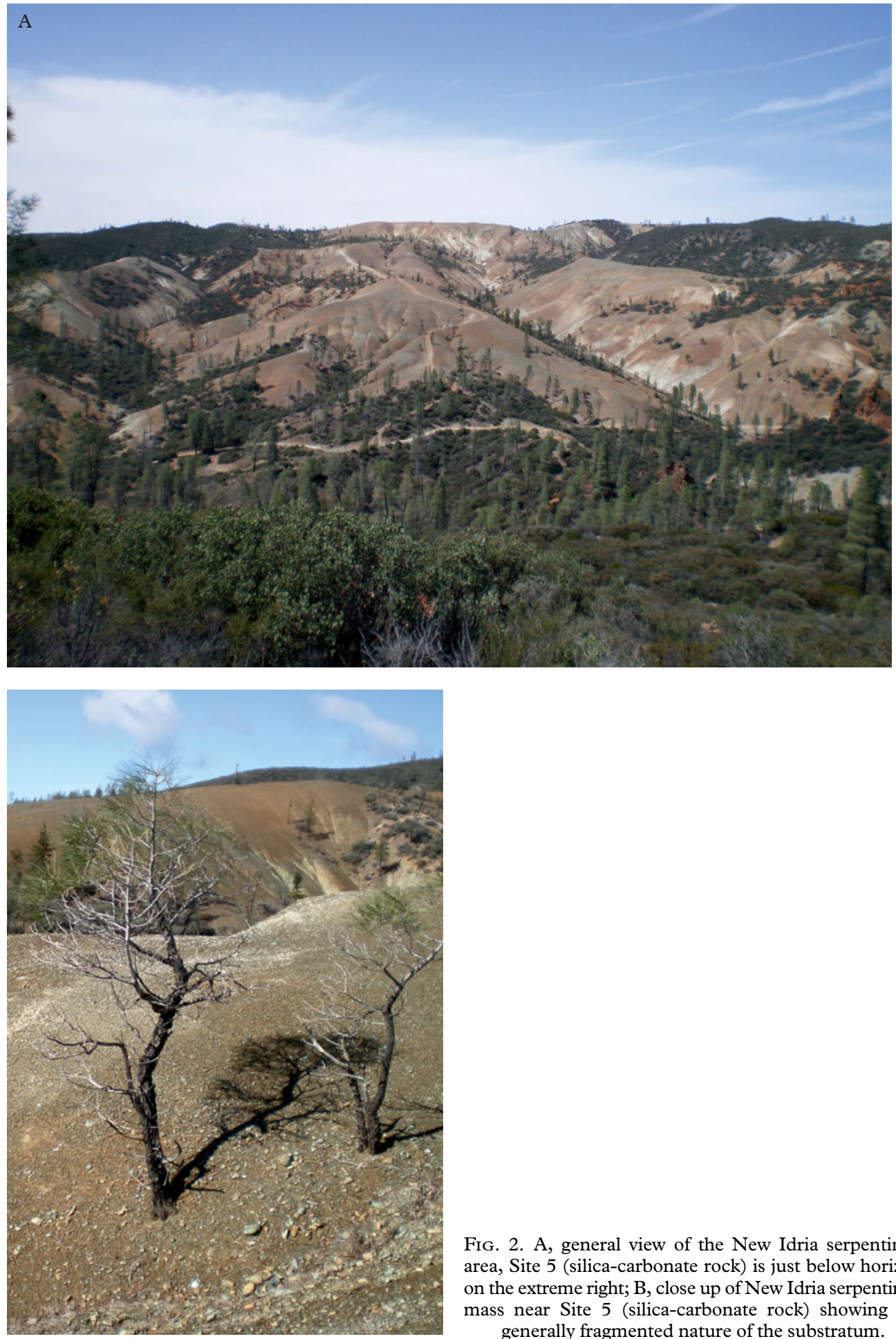

FIg. 2. A, general view of the New Idria serpentinite area, Site 5 (silica-carbonate rock) is just below horizon on the extreme right; B, close up of New Idria serpentinite mass near Site 5 (silica-carbonate rock) showing the generally fragmented nature of the substratum. 

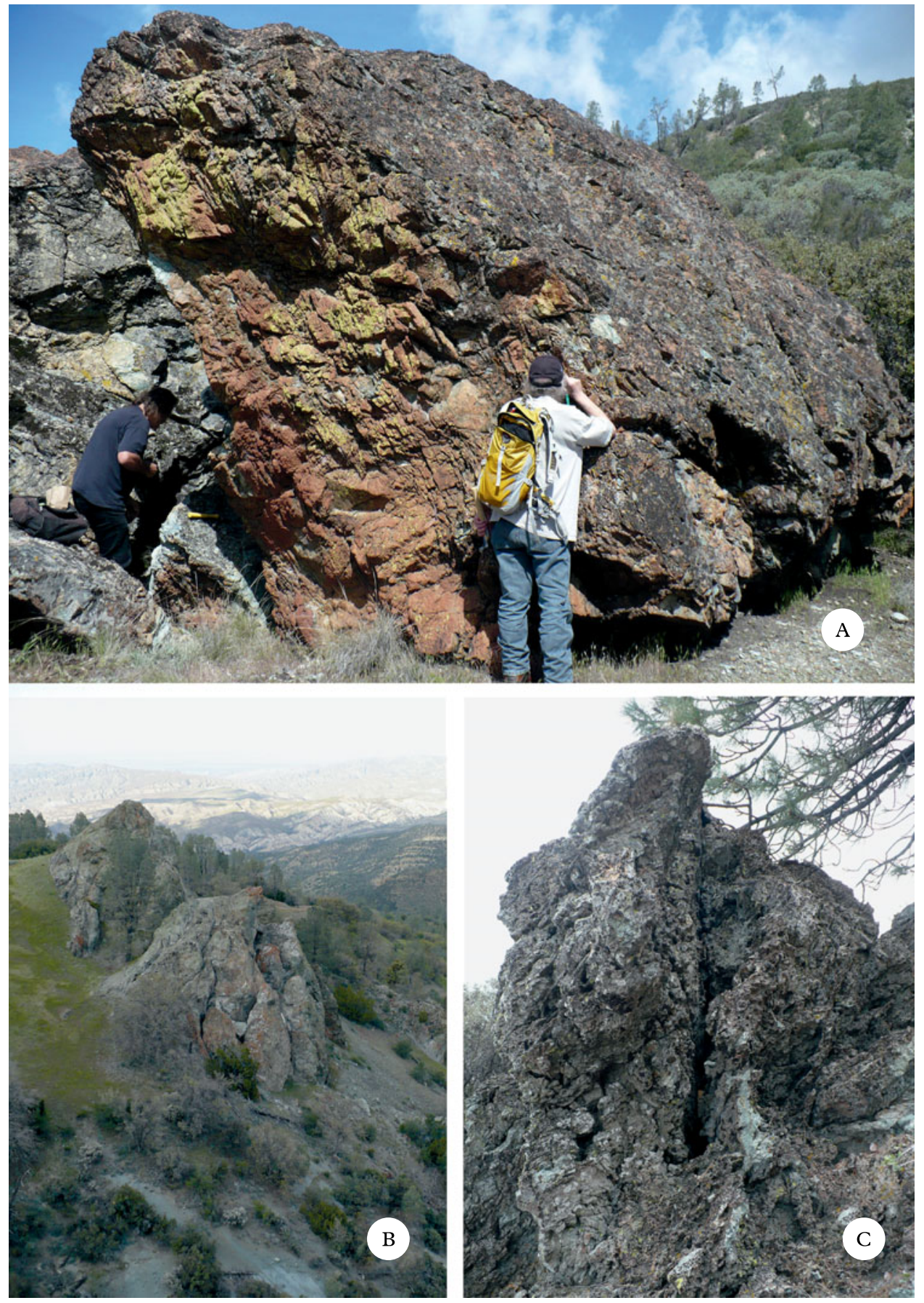

FIG. 3. Ultramafic outcrops. A, nephrite: large boulder at Staging Area (Site 1); B, serpentinite: over view of New Idria Reservoir (Site 6); C, serpentinite: close-up of a small part of San Benito Mountain Summit (Site 10). 

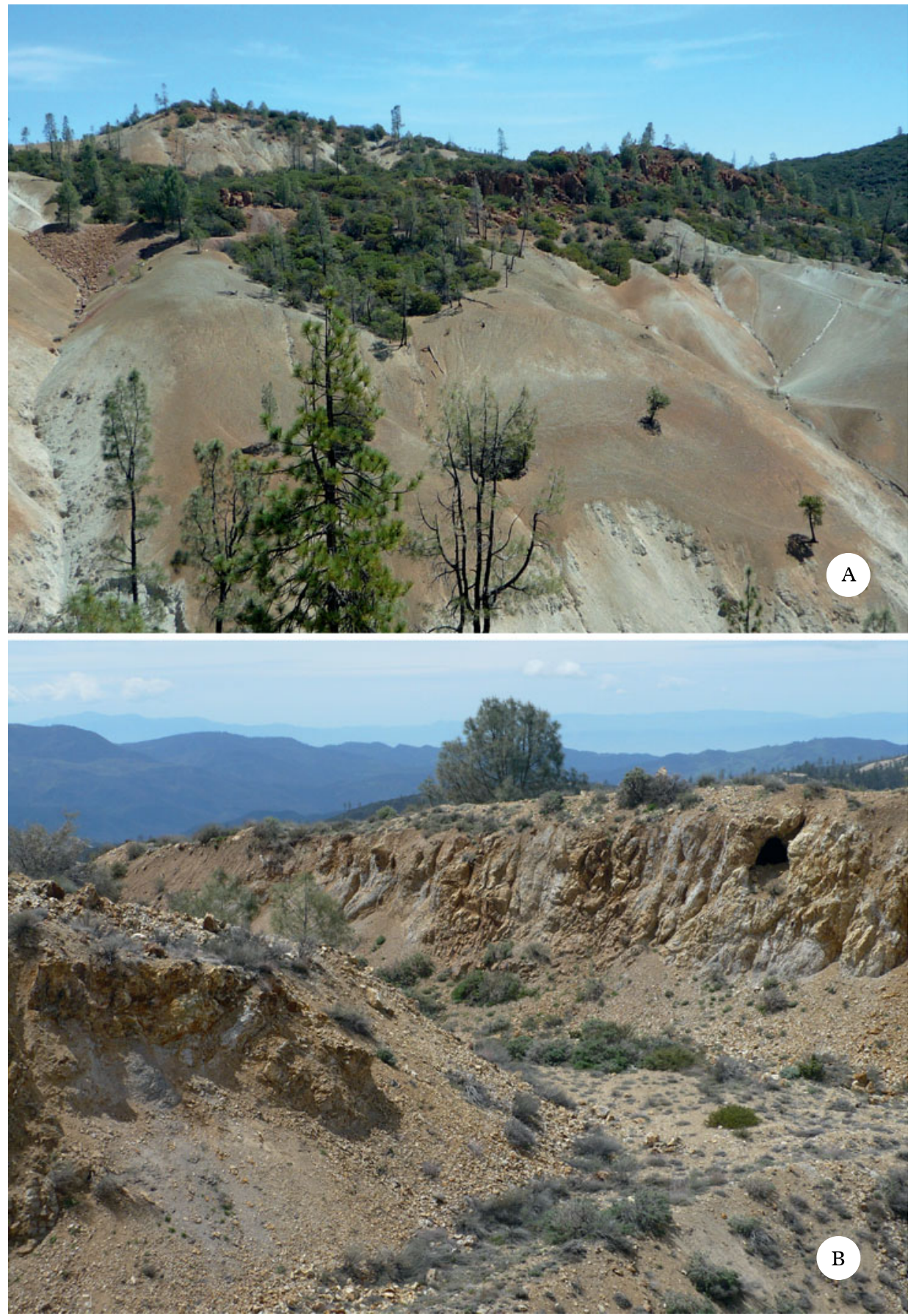

FIG. 4. Non-ultramafic rock types studied. A, silica-carbonate: Clear Creek Mine just below horizon (Site 5); B, shale and sandstone: San Carlos Peak Mine (Site 9). 
TABLE 1. Locality and substratum information for Sites 1-10 from which lichens were collected

\begin{tabular}{|c|c|c|c|c|c|c|c|c|c|}
\hline Site & Locality & Coordinates & $\begin{array}{l}\text { Elevation } \\
\quad(\mathrm{m})\end{array}$ & Substratum & $\begin{array}{l}\text { Rock } \\
\text { surface } \\
\text { texture }\end{array}$ & $\begin{array}{l}\text { Dominant } \\
\text { slope } \\
\text { aspect }\end{array}$ & $\begin{array}{c}\text { Disturbance } \\
\text { to rock } \\
\text { surface }\end{array}$ & $\begin{array}{l}\text { Sampling } \\
\text { area }\left(\mathrm{m}^{2}\right)\end{array}$ & $\begin{array}{l}\text { Total } \\
\text { area } \\
\left(\mathrm{m}^{2}\right)\end{array}$ \\
\hline 1 & Staging Area 1 & $\begin{array}{l}36^{\circ} 22^{\prime} 12.22^{\prime \prime} \mathrm{N} \\
120^{\circ} 44^{\prime} 51.63^{\prime \prime} \mathrm{W}\end{array}$ & 843 & Nephrite & $\begin{array}{l}\text { Fine granular } \\
\text { to lamellar }\end{array}$ & South & None & 59 & 59 \\
\hline 2 & $\begin{array}{l}\text { Between Staging } \\
\text { Area } 1 \text { and } 2\end{array}$ & $\begin{array}{l}36^{\circ} 22^{\prime} 12.08^{\prime \prime} \mathrm{N} \\
120^{\circ} 44^{\prime} 42.16^{\prime \prime} \mathrm{W}\end{array}$ & 835 & Nephrite & $\begin{array}{l}\text { Fine granular } \\
\text { to lamellar }\end{array}$ & North-west & None & 34 & 34 \\
\hline 3 & $\begin{array}{l}\text { Clear Creek Road } \\
\text { below Lower } \\
\text { Silica-Carbonate } \\
\text { Outcrop }\end{array}$ & $\begin{array}{l}36^{\circ} 22^{\prime} 31.60^{\prime \prime} \mathrm{N} \\
120^{\circ} 43^{\prime} 54.50^{\prime \prime} \mathrm{W}\end{array}$ & 914 & $\begin{array}{l}\text { Partially } \\
\text { serpentinized } \\
\text { peridotite }\end{array}$ & Coarse granular & South & None & 9 & 9 \\
\hline 4 & $\begin{array}{l}\text { Lower Silica- } \\
\text { Carbonate Outcrop }\end{array}$ & $\begin{array}{l}36^{\circ} 22^{\prime} 41.79^{\prime \prime} \mathrm{N} \\
120^{\circ} 43^{\prime} 40.06^{\prime \prime} \mathrm{W}\end{array}$ & 955 & $\begin{array}{l}\text { Silica- } \\
\text { Carbonate }\end{array}$ & Vacuolar porous & South-west & $\begin{array}{l}\text { Mining } \\
\text { (ceased } \\
\sim 1950 \text { ?) }\end{array}$ & 360 & 19456 \\
\hline 5 & $\begin{array}{l}\text { Upper Silica- } \\
\text { Carbonate Outcrop }\end{array}$ & $\begin{array}{l}36^{\circ} 22^{\prime} 48.07^{\prime \prime} \mathrm{N} \\
120^{\circ} 43^{\prime} 39.90^{\prime \prime} \mathrm{W}\end{array}$ & 1014 & $\begin{array}{l}\text { Silica- } \\
\text { Carbonate }\end{array}$ & Vacuolar porous & South-west & $\begin{array}{l}\text { Mining } \\
\text { (ceased } \\
\sim 1950 \text { ?) }\end{array}$ & 403 & 19456 \\
\hline 6 & New Idria Reservoir & $\begin{array}{l}36^{\circ} 24^{\prime} 5.65^{\prime \prime} \mathrm{N} \\
120^{\circ} 40^{\prime} 30.82^{\prime \prime} \mathrm{W}\end{array}$ & 1164 & Serpentinite & Lamellar & West & None & 89 & 303 \\
\hline 7 & $\begin{array}{l}\text { New Idria Mine } \\
\text { Tailings }\end{array}$ & $\begin{array}{l}36^{\circ} 24^{\prime} 55.47^{\prime \prime} \mathrm{N} \\
120^{\circ} 40^{\prime} 21.81^{\prime \prime} \mathrm{W}\end{array}$ & 812 & $\begin{array}{l}\text { Shale and } \\
\text { Sandstone } \\
\text { (furnace tailings) }\end{array}$ & $\begin{array}{l}\text { Clastic; medium } \\
\text { to coarse grained }\end{array}$ & North-east & $\begin{array}{l}\text { Mining } \\
\text { (ceased } \\
\sim 1972 \text { ) }\end{array}$ & 1833 & 156191 \\
\hline 8 & $\begin{array}{l}\text { New Idria Camp } \\
2 \text { Pit }\end{array}$ & $\begin{array}{l}36^{\circ} 24^{\prime} 33.42^{\prime \prime} \mathrm{N} \\
120^{\circ} 40^{\prime} 52.60^{\prime \prime} \mathrm{W}\end{array}$ & 1087 & $\begin{array}{l}\text { Shale and } \\
\text { Sandstone }\end{array}$ & $\begin{array}{l}\text { Clastic; medium } \\
\text { to coarse grained }\end{array}$ & North & $\begin{array}{l}\text { Mining } \\
\text { (ceased } \\
\sim 1972 \text { ) }\end{array}$ & 555 & 154072 \\
\hline 9 & $\begin{array}{l}\text { San Carlos Peak } \\
\text { Mine Pit }\end{array}$ & $\begin{array}{l}36^{\circ} 23^{\prime} 34.14^{\prime \prime} \mathrm{N} \\
120^{\circ} 39^{\prime} 31.17^{\prime \prime} \mathrm{W}\end{array}$ & 1426 & $\begin{array}{l}\text { Shale and } \\
\text { Sandstone }\end{array}$ & $\begin{array}{l}\text { Clastic; medium to } \\
\text { coarse grained }\end{array}$ & South & $\begin{array}{l}\text { Mining } \\
\text { (ceased } \\
\sim 1972 \text { ) }\end{array}$ & 6694 & 66264 \\
\hline 10 & $\begin{array}{l}\text { San Benito } \\
\text { Mountain Summit }\end{array}$ & $\begin{array}{l}36^{\circ} 22^{\prime} 10.49^{\prime \prime} \mathrm{N} \\
120^{\circ} 38^{\prime} 40.82^{\prime \prime} \mathrm{W}\end{array}$ & 1602 & Serpentinite & Vacuolar porous & South & None & 42 & 433 \\
\hline
\end{tabular}


parameters method. Approximately 105 diverse certified reference materials were employed for instrument calibration, and two internal standards were run on a regular basis to provide a continuous check on instrument performance.

\section{Statistical analysis}

Multiple permutational one-way ANOVAs with 999 permutations (Legendre 2007) and post-hoc comparisons implemented by package coin (Hothorn et al. 2008) were used to test the hypothesis that measured elemental concentrations differed across rocks collected from nephrite + partially serpentinized peridotite + serpentinite (collectively 'ultramafic'), silica-carbonate, and shale and sandstone sites (collectively 'non-ultramafic'). A Benjamini-Hotchberg correction for multiple comparisons was applied to the $P$-values from these 32 variables to control for false discovery rates (FDR), which is suitable for situations where explanatory variables are correlated between multiple tests (García 2003). A $t$-test was used to compare log-transformed species richness per $10 \mathrm{~m} \times 10 \mathrm{~m}$ sampling area between ultramafic and nonultramafic sites. A permutational multivariate analysis of variance (perMANOVA) with 999 permutations, function adonis of package vegan (Oksanen et al. 2011) was used to compare the assemblage of lichens among ultramafic vs. non-ultramafic sites. This comparison was chosen based upon the substantial differences in elemental composition between the two rock types. The sizes of the matrices included in the perMANOVA were 10 sites by 112 species and 44 genera. Lichenicolous fungi were excluded from the analysis because they are mostly species-specific and including them in the analysis would be equivalent to including their host species twice. Function adonis uses a dissimilarity matrix to statistically compare the squared deviations of multivariate group centroids, and is well suited to the analysis of biotic community assemblage where the presence or absence of many taxa must be compared across few regions (Anderson 2001; McArdle \& Anderson 2001). Equal dispersion of group scores (analogous to a test for multivariate homogeneity of variances) was assessed using function betadisper in package vegan (Oksanen et al. 2011). Kulczynski distance (Faith et al. 1987) was chosen as an appropriate index of dissimilarity as it is robust to 'richness dependency', where site pairs with similar composition but differing richness receive high dissimilarity values (Hausdorf \& Hennig 2005). To check for correlation between patterns of community assemblage and substratum elemental composition, a Mantel test (with 999 permutations; function mantel in package vegan) was employed using a Kulczynski distance matrix of beta diversity and a matrix of variancescaled, mean-centred Euclidean distances for the correlated elemental variables. All statistical analyses were performed using $\mathrm{R}$ version 2.13.2 ( $\mathrm{R}$ Development Core Team 2011).

\section{Results}

\section{Rock chemistry}

The composition of measured ultramafic, silica-carbonate, and shale and sandstone rocks differed significantly for 26 of 32 elements (Table 2; permutational one-way ANOVA; Benjamini-Hotchberg corrected $P<0 \cdot 05$ ). Notable distinctions include significantly lower $\mathrm{Ca}: \mathrm{Mg}$ ratios for ultramafic rocks and higher concentrations of heavy metals such as Ni and Cr. Additionally, non-ultramafic rocks were significantly higher compared to ultramafic rocks in rare earth elements such as $\mathrm{Ba}, \mathrm{Rb}, \mathrm{Sr}, \mathrm{V}, \mathrm{Y}$, and $\mathrm{Zr}$.

\section{Floristics}

We identified a total of 119 species of lichenized and lichenicolous fungi (Table 3), of which four, Buellia ocellata, Caloplaca oblongula, Rhizocarpon saurinum, and Thelocarpon laureri, are reported for the first time from California, and two, Buellia aethalea and Trapelia obtegens, are represented from California only by unpublished collections in the Consortium of North American Lichen Herbaria database (http://symbiota.org/nalichens). Buellia aethalea was collected from ultramafic rocks, B. ocellata from ultramafic and nonultramafic rocks, and the other four species from non-ultramafic rocks. Additionally, a collection of a Solenopsora sp. from silicacarbonate rock does not correspond to any of the species of this genus currently listed as occurring in North America (Esslinger 2011), and is under further investigation by molecular methods to confirm its taxonomic status.

By far the largest number of taxa (83) was collected from ultramafic rocks, with the two other rock types sampled, silica-carbonate (37) and shale and sandstone (28) (nonultramafic rocks), being far less species-rich. A similar pattern is apparent for taxa collected from only one rock type, with ultramafic rocks (60) having far more taxa restricted to that substratum than the two non-ultramafic rock types: silica-carbonate (19), shale and 


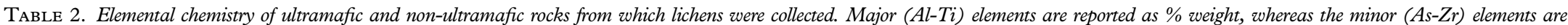

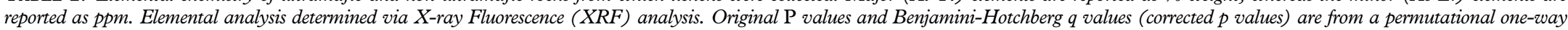

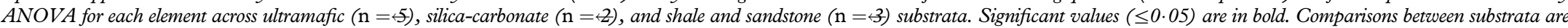
denoted with superscripted letters adjacent to their respective means \pm standard errors; different letters indicate a significant difference

\begin{tabular}{|c|c|c|c|c|c|c|c|c|c|c|c|c|c|c|}
\hline \multirow[b]{3}{*}{ Site\# $\rightarrow \leftarrow$} & \multicolumn{5}{|c|}{ Ultramafic rock } & \multicolumn{5}{|c|}{ Non-ultramafic rock } & \multirow{3}{*}{$\begin{array}{c}\text { Ultramafic } \\
\text { Nephrite; } \\
\text { Serpentinized } \\
\text { peridotite; } \\
\text { Serpentinite } \\
(\text { Mean } \pm \text { SE) }\end{array}$} & \multicolumn{2}{|c|}{ Non-ultramafic } & \multirow[b]{3}{*}{$P / \mathrm{q}$ Value } \\
\hline & \multicolumn{2}{|c|}{ Nephrite } & \multirow{2}{*}{$\frac{\begin{array}{c}\text { Serpentinized } \\
\text { peridotite }\end{array}}{3}$} & \multicolumn{2}{|c|}{ Serpentinite } & \multicolumn{2}{|c|}{ Silica-carbonate } & \multicolumn{3}{|c|}{$\begin{array}{l}\text { Shale and } \\
\text { sandstone }\end{array}$} & & \multirow[b]{2}{*}{$\begin{array}{l}\text { Silica-Carbonate } \\
\quad(\text { Mean } \pm S E)\end{array}$} & \multirow{2}{*}{$\begin{array}{l}\text { Shale and } \\
\text { Sandstone } \\
(\text { Mean } \pm S E)\end{array}$} & \\
\hline & 1 & 2 & & 6 & 10 & 4 & 5 & 7 & 8 & 9 & & & & \\
\hline \multicolumn{15}{|c|}{ Major Elements (\%) } \\
\hline $\mathrm{Al}_{2} \mathrm{O}_{3}$ & 0.26 & 0.28 & $4 \cdot 48$ & $1 \cdot 47$ & 0.22 & $15 \cdot \theta 4$ & $15 \cdot 44$ & $12 \cdot 46$ & $13 \cdot 64$ & $15 \cdot 48$ & $1 \cdot 28 \pm 0.82^{\mathbf{A}}$ & $15 \cdot 24 \pm 0 \cdot 2^{\mathbf{B}}$ & $13 \cdot 86 \pm 0.88^{\mathbf{B}}$ & $0.005 / 0.011$ \\
\hline $\mathrm{CaO}$ & 0.53 & 0 & 0.04 & 0.85 & 0.02 & 0.43 & $0 \cdot 41$ & $0 \cdot 48$ & $0 \cdot 42$ & 0.26 & $0.28 \pm 0.17$ & $0.12 \pm 0 \cdot \theta 1$ & $0.49 \pm 0.04$ & $0.855 / 0.883$ \\
\hline $\mathrm{FeO}$ & 4.95 & $5 \cdot 38$ & $8 \cdot 21$ & $6 \cdot 4$ & 8.64 & 0.2 & $7 \cdot 6$ & $4 \cdot 27$ & $5 \cdot 9$ & 3.69 & $6.66 \pm 0.75$ & $3 \cdot 9 \pm 3 \cdot 7$ & $4.62 \pm 0.66$ & $0.330 / 0.364$ \\
\hline $\mathrm{K}_{2} \mathrm{O}$ & 0.03 & $0 \cdot 01$ & $0 \cdot \theta 1$ & 0 & 0.01 & $2 \cdot 41$ & 2.69 & 6.99 & 1.67 & 3.33 & $0 \cdot \theta 1 \pm 0 . \theta 04^{\mathbf{A}}$ & $2 \cdot 55 \pm 0.44^{\mathbf{B}}$ & $4 \cdot 00 \pm 1 \cdot 57^{\mathbf{B}}$ & $0.011 / 0.017$ \\
\hline $\mathrm{MgO}$ & $35 \cdot 2$ & $38 \cdot 79$ & 34.92 & $37 \cdot 25$ & $31 \cdot 46$ & 0.28 & 0.29 & 0.25 & 0.23 & $0 \cdot 41$ & $35.52 \pm 1.24^{\mathbf{A}}$ & $0.29 \pm 0.005^{\mathbf{B}}$ & $0.30 \pm 0.06^{\mathbf{B}}$ & $0.012 / 0.017$ \\
\hline $\mathrm{CaO}: \mathrm{MgO}$ & 0.015 & 0 & 0.001 & 0.023 & 0.001 & 0.461 & 0.377 & 0.734 & 0.52 & 0.629 & $0.008 \pm 0.005^{\mathbf{A}}$ & $0.419 \pm 0.042^{\mathbf{B}}$ & $0.628 \pm 0.062^{\mathbf{B}}$ & $<0.001 / 0.005$ \\
\hline $\mathrm{MnO}$ & $0 \cdot 408$ & 0.098 & $0 \cdot 467$ & $0 \cdot 411$ & 0.098 & 0.002 & 0 & 0.082 & 0.02 & 0.016 & $0.416 \pm 0.013^{\mathbf{A}}$ & $0.001 \pm 0.001^{\mathbf{B}}$ & $0.039 \pm 0.021^{\mathbf{B}}$ & $<0.001 / 0.005$ \\
\hline $\mathrm{Na}_{2} \mathrm{O}$ & $0 . \theta 4$ & $0 . \theta 1$ & 0 & 0 & $0 . \theta 1$ & $4 \cdot 61$ & $6 \cdot 03$ & $1 \cdot 4$ & $6 \cdot 47$ & $5 \cdot 23$ & $0 \cdot \theta 1 \pm 0 . \theta 07^{\mathbf{A}}$ & $5 \cdot 32 \pm 0.71^{\mathbf{B}}$ & $4 \cdot 47 \pm 1 \cdot 56^{\mathbf{B}}$ & $0.008 / 0.015$ \\
\hline $\mathrm{P}_{2} \mathrm{O}_{5}$ & 0.017 & 0.007 & 0.007 & 0.005 & 0.01 & 0.041 & $0 \cdot 4$ & $0 \cdot 441$ & 0.09 & $0 \cdot 418$ & $0.009 \pm 0.002^{\mathbf{A}}$ & $0.071 \pm 0.03^{\mathbf{B}}$ & $0 \cdot 416 \pm 0 \cdot 015^{\mathbf{B}}$ & $<0.001 / 0.005$ \\
\hline $\mathrm{SiO}_{2}$ & $16 \cdot 63$ & $43 \cdot 01$ & 39.88 & 41.62 & $48 \cdot \theta 8$ & $72 \cdot 43$ & $61 \cdot 25$ & $70 \cdot 54$ & 64.52 & 66.99 & $37 \cdot 84 \pm 5 \cdot 48^{\mathbf{A}}$ & $66 \cdot 69 \pm 5 \cdot 44^{\mathbf{B}}$ & $67 \cdot 35 \pm 1.75^{\mathbf{B}}$ & $0.007 / 0.014$ \\
\hline $\mathrm{SO}_{3}$ & 0.02 & 0 & 0 & 0 & 0 & 0 & 0.31 & 0 & $1 \cdot 42$ & 0 & $0 \cdot \theta 04 \pm 0 \cdot \theta 04$ & $0 \cdot 455 \pm 0 \cdot 155$ & $0.473 \pm 0.473$ & $0.353 / 0 \cdot 377$ \\
\hline $\mathrm{TiO}_{2}$ & $0 \cdot 017$ & $0 \cdot \theta \theta 2$ & 0.016 & $0 \cdot 008$ & 0.003 & 0.702 & 0.71 & 0.604 & 0.65 & 0.673 & $0.009 \pm 0.003^{\mathbf{A}}$ & $0.706 \pm 0.04^{\mathbf{B}}$ & $0.642 \pm 0.02^{\mathbf{B}}$ & $0.002 / 0.0 \theta 7$ \\
\hline \multicolumn{15}{|c|}{ Trace Elements (ppm) } \\
\hline As & 0 & 3 & 0 & 0 & 0 & 0 & 30 & 15 & 5 & 9 & $0 \pm 0^{\mathbf{A}}$ & $15 \pm 15^{\mathbf{A}, \mathbf{B}}$ & $9 \cdot 7 \pm 2 \cdot 9^{\mathbf{B}}$ & $0.072 / 0.085$ \\
\hline $\mathrm{Ba}$ & 36 & 7 & 28 & 16 & 7 & 501 & 608 & 831 & 414 & 850 & $18 \cdot 8 \pm 5 \cdot 8^{\mathbf{A}}$ & $554 \cdot 5 \pm 53 \cdot 5^{\mathbf{B}}$ & $698 \cdot 3 \pm 142 \cdot 3^{\mathbf{B}}$ & $<0.001 / 0.005$ \\
\hline $\mathrm{Ce}$ & 3 & 0 & 0 & 3 & 0 & 42 & 34 & 21 & 28 & 45 & $1 \cdot 2 \pm 0 \cdot 7^{\mathbf{A}}$ & $38 \pm 4^{\mathbf{B}}$ & $31 \cdot 3 \pm 7 \cdot 4^{\mathbf{B}}$ & $0.002 / 0.007$ \\
\hline $\mathrm{Cr}$ & 2766 & 2381 & 935 & 1971 & 999 & 104 & 115 & 112 & 111 & 103 & $1810 \pm 367^{\mathbf{A}}$ & $110 \pm 6^{\mathbf{B}}$ & $109 \pm 3^{\mathbf{B}}$ & $0.028 / 0.036$ \\
\hline $\mathrm{Cu}$ & 7 & 3 & 3 & 8 & 3 & 8 & 54 & 112 & 62 & 52 & $4 \cdot 8 \pm 1 \cdot 4^{\mathbf{A}}$ & $31 \pm 23^{\mathbf{A}, \mathbf{B}}$ & $75 \cdot 3 \pm 18 \cdot 6^{\mathbf{B}}$ & $0.012 / 0.017$ \\
\hline $\mathrm{Ga}$ & 1 & 1 & 4 & 2 & 1 & 15 & 19 & 15 & 14 & 18 & $1.8 \pm 0.6^{\mathbf{A}}$ & $17 \pm 2^{\mathbf{B}}$ & $15 \cdot 7 \pm 1 \cdot 2^{\mathbf{B}}$ & $0.004 / 0 \cdot \theta 10$ \\
\hline $\mathrm{La}$ & 2 & 4 & 0 & 1 & 1 & 21 & 21 & 12 & 12 & 24 & $1.6 \pm 0.7^{\mathbf{A}}$ & $21 \pm 0^{\mathbf{B}}$ & $16 \pm 4^{\mathbf{B}}$ & $<0.001 / 0.005$ \\
\hline $\mathrm{Nb}$ & $0 \cdot 4$ & 0.7 & 0.7 & 0.6 & $0 \cdot 3$ & $9 \cdot 4$ & $9 \cdot 5$ & $5 \cdot 9$ & 9 & $8 \cdot 4$ & $0.54 \pm 0.08^{\mathbf{A}}$ & $9 \cdot 45 \pm 0.05^{\mathbf{B}}$ & $7 \cdot 77 \pm 0.95^{\mathbf{B}}$ & $<0.001 / 0.005$ \\
\hline $\mathrm{Nd}$ & 2 & 1 & 1 & 3 & 1 & 17 & 13 & 14 & 11 & 20 & $1 \cdot 6 \pm 0 \cdot 4^{\mathrm{A}}$ & $15 \pm 2^{\mathbf{B}}$ & $15 \pm 2 \cdot 6^{\mathbf{B}}$ & $0.009 / 0.015$ \\
\hline $\mathrm{Ni}$ & 1839 & 1912 & 2074 & 1933 & 2662 & 22 & 17 & 105 & 22 & 115 & $2084 \pm 149^{\mathbf{A}}$ & $20 \pm 3^{\mathbf{B}}$ & $81 \pm 29^{\mathbf{B}}$ & $0 \cdot 010 / 0 \cdot \theta 16$ \\
\hline $\mathrm{Pb}$ & 1 & 3 & 42 & 1 & 3 & 12 & 8 & 10 & 10 & 17 & $10 \pm 8$ & $10 \pm 2$ & $12 \pm 2$ & $0.988 / 0.988$ \\
\hline $\mathrm{Rb}$ & 1 & 0 & 1 & 0 & 0 & 63 & 85 & 172 & 53 & 94 & $0 \cdot 4 \pm 0 \cdot 2^{\mathbf{A}}$ & $74 \pm 11^{\mathbf{B}}$ & $106 \cdot 3 \pm 34 \cdot 9^{\mathbf{B}}$ & $0 \cdot \theta \theta 2 / 0 \cdot \theta \theta 7$ \\
\hline Sc & 6 & 5 & 13 & 8 & 5 & 9 & 10 & 22 & 15 & 17 & $7 \pm 1 \cdot 5^{\mathbf{A}}$ & $9 \cdot 5 \pm 0 \cdot 5^{\mathbf{A}, \mathbf{B}}$ & $18 \pm 2^{\mathbf{B}}$ & $0 \cdot 009 / 0 \cdot 015$ \\
\hline $\mathrm{Sr}$ & 40 & 1 & 4 & 74 & 1 & 77 & 74 & 62 & 125 & 86 & $24 \pm 14 \cdot 5^{\mathbf{A}}$ & $75 \cdot 5 \pm 1 \cdot 5^{\mathbf{A}, \mathbf{B}}$ & $91 \pm 18 \cdot 4^{\mathbf{B}}$ & $0.041 / 0.050$ \\
\hline $\mathrm{Th}$ & 0 & 0 & 0 & 0 & 0 & 5 & 8 & 4 & 4 & 8 & $0 \pm 0^{\mathbf{A}}$ & $6 \cdot 5 \pm 1 \cdot 5^{\mathbf{B}}$ & $5 \cdot 3 \pm 1 \cdot 3^{\mathbf{B}}$ & $0 \cdot \theta 04 / 0 \cdot \theta 10$ \\
\hline $\mathrm{U}$ & 0 & 0 & 1 & 0 & 0 & 1 & 4 & 2 & 2 & 2 & $0 . \mathbf{2} \pm 0.2^{\mathbf{A}}$ & $2 \cdot 5 \pm 1 \cdot 5^{\mathbf{A}, \mathbf{B}}$ & $2 \pm 0^{\mathbf{B}}$ & $0.017 / 0.023$ \\
\hline $\mathrm{V}$ & 22 & 25 & 62 & 38 & 10 & 128 & 133 & 142 & 126 & 137 & $31 \cdot 4 \pm 8 \cdot 9^{\mathbf{A}}$ & $130 \cdot 5 \pm 2 \cdot 5^{\mathbf{B}}$ & $135 \pm 4 \cdot 7^{\mathbf{B}}$ & $0 \cdot 004 / 0 \cdot \theta 10$ \\
\hline $\mathrm{Y}$ & 2 & 2 & $\begin{array}{r}02 \\
2\end{array}$ & 2 & 1 & $\begin{array}{r}120 \\
22\end{array}$ & 19 & $\begin{array}{r}142 \\
22\end{array}$ & 13 & 21 & $1 \cdot 8 \pm 0 \cdot 2^{\mathbf{A}}$ & $20 \cdot 5 \pm 1.5^{\mathbf{B}}$ & $18 \cdot 7 \pm 2 \cdot 8^{\mathbf{B}}$ & $0.005 / 0.011$ \\
\hline $\mathrm{Zn}$ & 38 & 40 & 51 & 41 & 31 & 4 & 25 & 113 & 17 & 82 & $40 \cdot 2 \pm 3 \cdot 2$ & $14.5 \pm 10.5$ & $70 \cdot 7 \pm 28 \cdot 3$ & $0 \cdot 403 / 0.418$ \\
\hline $\mathrm{Zr}$ & 5 & 0 & 4 & 0 & 1 & 138 & 160 & 105 & 144 & 135 & $2 \pm 1 \cdot 4^{\mathbf{A}}$ & $149 \pm 11^{\mathbf{B}}$ & $128 \pm 11 \cdot 8^{\mathbf{B}}$ & $0 \cdot 004 / 0 \cdot \theta 10$ \\
\hline
\end{tabular}


TABLE 3. 112 lichen taxa and 7 lichenicolous fungi collected from 10 sites at the New Idria serpentinite mass. The six taxa in bold font are new reports or newly published records for California. Names marked with a ${ }^{*}$ were reported from more than one ultramafic site by FaveroLongo et al. (2004), and those with a † by Sigal (1989). Nomenclature and naming authorities follow Index Fungorum Partnership (http://indexfungorum.org)

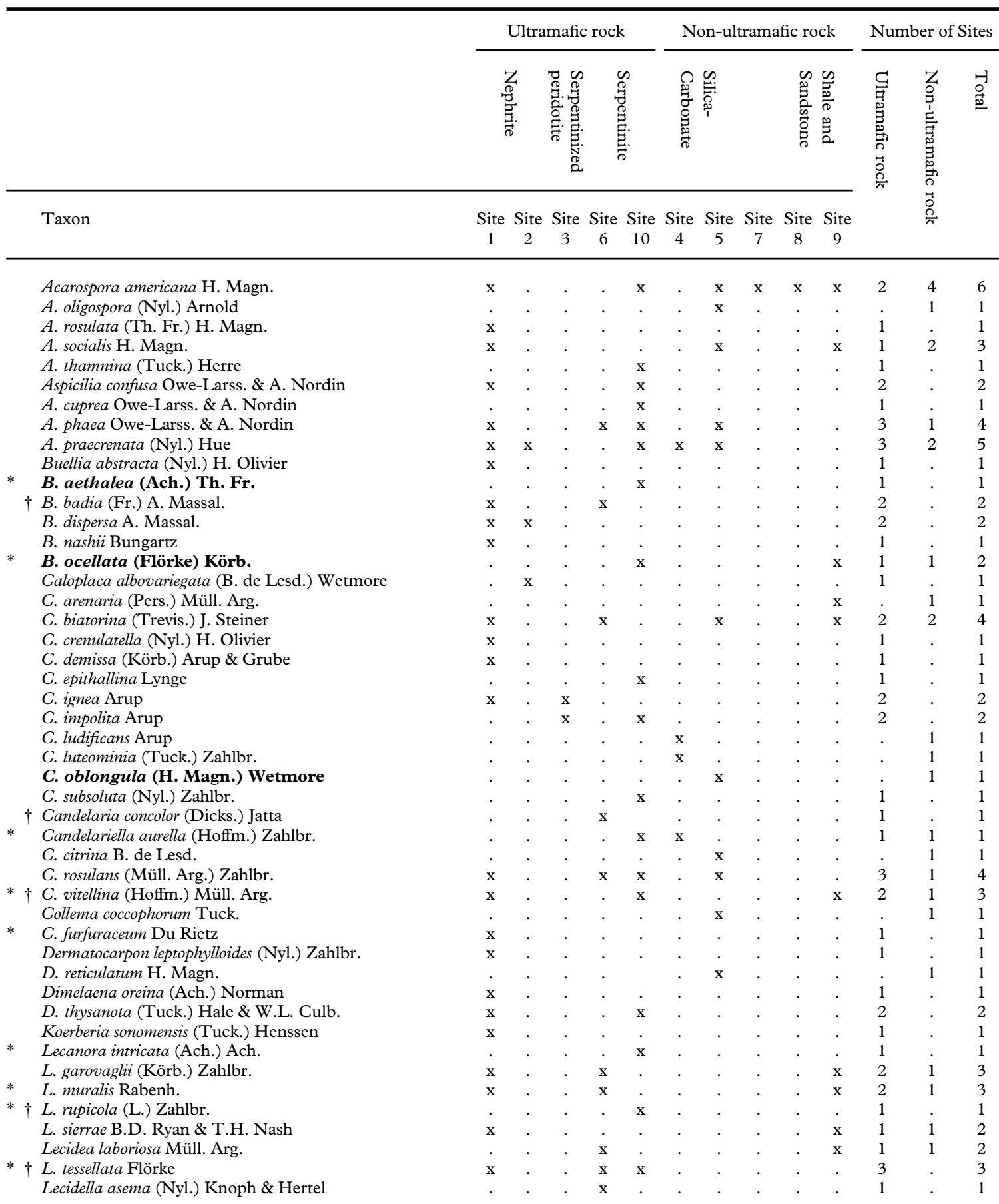


TABle 3. Continued

\begin{tabular}{|c|c|c|c|c|c|c|c|c|c|}
\hline & \multicolumn{3}{|c|}{ Ultramafic rock } & \multicolumn{3}{|c|}{ Non-ultramafic rock } & \multicolumn{3}{|c|}{ Number of Sites } \\
\hline & 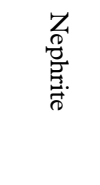 & 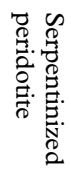 & 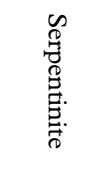 & 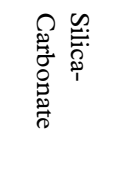 & & 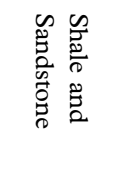 & \multirow[t]{2}{*}{$\begin{array}{l}\text { S } \\
\overrightarrow{7} \\
\stackrel{0}{0} \\
\stackrel{0}{0} \\
\stackrel{0}{0} \\
\overrightarrow{0} \\
\stackrel{0}{\pi}\end{array}$} & \multirow{2}{*}{ 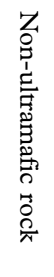 } & \multirow{2}{*}{$\begin{array}{l}\text { 멍 } \\
\stackrel{0}{0}\end{array}$} \\
\hline Taxon & $\begin{array}{cc}\text { Site } & \text { Site } \\
1 & 2\end{array}$ & $\begin{array}{c}\text { Site } \\
3\end{array}$ & $\begin{array}{cc}\text { Site } & \text { Site } \\
6 & 10\end{array}$ & $\begin{array}{cc}\text { Site } & \text { Site } \\
4 & 5\end{array}$ & $\begin{array}{c}\text { Site } \\
7\end{array}$ & $\begin{array}{cc}\text { Site } & \text { Site } \\
8 & 9\end{array}$ & & & \\
\hline
\end{tabular}

$* \dagger$ Lecidella carpathica Körb.

* $\dagger$ L. stigmatea (Ach.) Hertel \& Leuckert

Lepraria texta $\mathrm{K}$. Knudsen et al.

$\dagger$ Leptochidium albociliatum (Desm.) M. Choisy

$\dagger$ Leptogium californicum Tuck.

L. lichenoides (L.) Zahlbr.

* L. tenuissimum (Hoffm.) Körb.

Lichenothelia tenuissima Henssen

Melanohalea elegantula (Zahlbr.) O. Blanco et al.

Melanelixia glabroides (Essl.) O. Blanco et al.

Myriospora scabrida (H. Magn.) K. Knudsen \& L. Arcadia

$\dagger$ Peltula bolanderi (Tuck.) Wetmore

P. euploca (Ach.) Poelt ex Ozenda \& Clauzade

Physcia biziana (A. Massal.) Zahlbr.

P. stellaris (L.) Nyl.

$P$. dimidiata (Arnold) Nyl.

Physconia americana Essl.

P. californica Essl.

P. enteroxantha (Nyl.) Poelt

$P$. isidiomuscigena Essl.

* P. muscigena (Ach.) Poelt

Placidium lacinulatum (Ach.) Breuss

P. squamulosum (Ach.) Breuss

Placopyrenium stanfordii (Herre) K. Knudsen

Placynthiella hyporhoda (Th. Fr.) Coppins \& P. James

Polysporina simplex (Taylor) Jatta

Protoblastenia rupestris (Scop.) J. Steiner

Psora luridella (Tuck.) Fink

Psorotichia hassei Fink ex J. Hedrick

P. montinii (A. Massal.) Forssell

$\dagger$ Rhizocarpon bolanderi (Tuck.) Herre

$R$. disporum (Nägeli ex Hepp) Müll. Arg.

$*+R$. geographicum (L.) DC.

R. saurinum (W.A. Weber) Bungartz

$R$. superficiale (Schaer.) Malme

$* \dagger R$. viridiatrum (Wulfen) Körb.

Rhizoplaca glaucophana (Hasse) W.A. Weber

$\dagger R$. melanophthalma (DC.) Leuckert

Rinodina bischoffii (Hepp) A. Massal.

* $\quad$ R. confragosa (Ach.) Körb.

* R. gennarii Bagl.

R. milvina (Wahlenb.) Th. Fr.

R. obnascens (Nyl.) H. Olivier

$R$. straussii J. Steiner

Sarcogyne arenosa (Herre) K. Knudsen \& S.M. Standl.

Solenopsora sp.

Staurothele areolata (Ach.) Lettau

S. elenkinii Oxner

Thelocarpon laureri (Flot.) Nyl. 
TABle 3. Continued

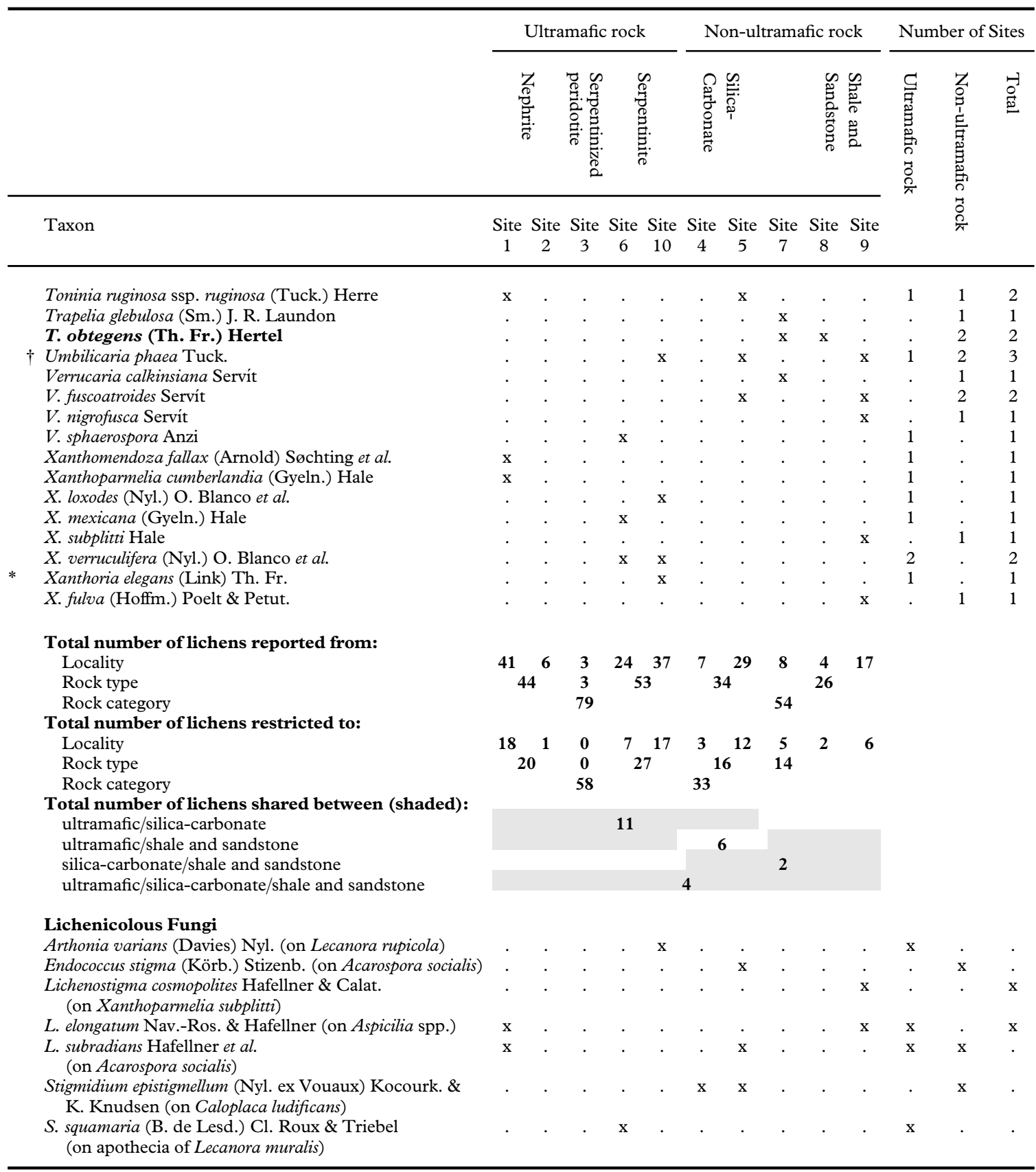


TABLE 4. Results from perMANOVA with 1000 permutations, showing differences in lichen community assembly between ultramafic and non-ultramafic (silica-carbonate and shale and sandstone) rocks at the species and generic level

\begin{tabular}{lcccc}
\hline & df & $F$ & $\mathrm{R}^{2}$ & $P$ value \\
\hline Species (112) & & & & \\
$\quad$ Substratum & 1 & $1 \cdot 812$ & $0 \cdot 185$ & $0 \cdot 020$ \\
Residuals & 8 & $\cdot$ & $0 \cdot 815$ & $\cdot$ \\
$\quad$ Total & 9 & $\cdot$ & & $\cdot$ \\
Genera (44) & & $1 \cdot 651$ & $0 \cdot 471$ & \\
$\quad$ Substratum & 1 & $\cdot$ & $0 \cdot 829$ & $\cdot 164$ \\
Residuals & 8 & $\cdot$ & 1 & $\cdot$ \\
$\quad$ Total & 9 & & & \\
\hline
\end{tabular}

sandstone (15). Data for taxa occurring on more than one substratum were ultramafic and silica-carbonate (11), ultramafic and shale and sandstone (6), silica-carbonate and shale and sandstone (2). Interestingly, only four species (Acaropsora americana, A. socialis, Caloplaca biatorina, and Umbilicaria phaea) occurred on all three rock types (Table 3).

\section{Lichen-substratum relations}

Species richness per $10 \mathrm{~m} \times 10 \mathrm{~m}$ sampling area was significantly greater at the ultramafic sites ( $t$-test; $t=\$ 5.51, P=40.002$; see Table $4)$, despite the wide range in species richness per site within each site group (Table 3), which was due, at least in part, to differences in the range of microhabitats present. Species richness standardized by area surveyed may not be an entirely accurate measure of alphadiversity, as species-area curves are asymptotic. However, undisturbed ultramafic areas had a much greater species richness than disturbed non-ultramafic areas, despite the much smaller average size of the former (Table 1). The perMANOVA revealed significant differences in lichen assemblage between ultramafic and non-ultramafic sites at the species level ( $P=0.020,112$ variables) but not at the generic level $(P=+0 \cdot 164,44$ variables; see Table 5). Dispersion of group scores was equal between ultramafic and nonultramafic sites $\left(P=0.683, \mathrm{H}_{0}=\right.$ no difference between groups). Species richness per $10 \mathrm{~m} \times 40 \mathrm{~m}$ sampling area and site scores from the species-level perMANOVA indicate that silica-carbonate sites supported lichen communities intermediate between ultramafic and shale and sandstone (see Table 6). However, we did not include silica-carbonate as a separate factor in our analysis due to small sample size. The most useful taxa in distinguishing groups by the perMANOVA are summarized in Table 6 . Lichen species assemblage and elemental composition among sites were weakly correlated (Mantel test; $r=\leftrightarrow \cdot 273, P=\leftarrow \theta \cdot 02)$.

\section{Discussion}

The importance of rock mineralogy, including elemental geochemistry, in determining the composition of saxicolous lichen communities has long been recognized (Purvis \& Halls 1996). However, as pointed out by Brodo (1973), attempts to analyze the distribution of saxicolous lichens according to their lithochemistry are not very common (e.g. Werner 1956), and studies that directly associate quantitatively assessed mineralogy or elemental chemistry of host rocks to the presence of lichen species or the assemblage of lichen communities are rare (e.g. Boyle et al. 1987). The exact nature of this substratum-level influence on lichens (i.e. whether chemical and/or textural) also appears to be obscure, although complex interactions between lichens and rocks and lichens and elements are often cited (Richardson 1995; Wilson 1995; Purvis 1996; Shimizu 2004; Hauck et al. 2007). Purvis (1996) states that systematic description of lichen communities 
TABLE 5. Species and genera that contribute substantially (absolute scores above the 95th percentile) to distinguishing ultramafic and non-ultramafic lichen communities in the perMANOVA model. 'Score' is the relative weight given to the taxon by the analysis. 'Occurrence' lists the sites where a given taxon occurred, with cross-over between substrata in bold font and, for genera, number of subtaxa given in parentheses

\begin{tabular}{|c|c|c|}
\hline Taxon & Score & Occurrence \\
\hline \multicolumn{3}{|l|}{ Ultramafic } \\
\hline \multicolumn{3}{|l|}{ Genus } \\
\hline Buellia & $0 \cdot 3$ & $1(4), 2(1), 4(1), 5(2), 10(1)$ \\
\hline Caloplaca & $0 \cdot 5$ & $1(4), 2(1), 3(2), 4(1), 5(3), 6(2), 7(2), 10(2)$ \\
\hline Lecanora & $0 \cdot 2$ & $1(3), 4(2), 5(2), 10(3)$ \\
\hline Leptogium & $0 \cdot 2$ & $1(2), 2(1), 7(1)$ \\
\hline Peltula & $0 \cdot 2$ & $1(2)$ \\
\hline Placopyrenium & $0 \cdot 2$ & $1(1), 3(1)$ \\
\hline \multicolumn{3}{|l|}{ Species } \\
\hline Aspicilia confusa & $0 \cdot 2$ & 1,10 \\
\hline A. phaea & $0 \cdot 2$ & $1,5,6,10$ \\
\hline Buellia badia & $0 \cdot 2$ & 1,6 \\
\hline B. dispersa & $0 \cdot 2$ & 1,2 \\
\hline Caloplaca ignea & $0 \cdot 2$ & 1,3 \\
\hline C. impolita & $0 \cdot 2$ & 3,10 \\
\hline Candelariella rosulans & $0 \cdot 2$ & $1,5,6,10$ \\
\hline Dimelaena thysanota & $0 \cdot 2$ & 1,10 \\
\hline Lecidea tessellata & $0 \cdot 3$ & $1,6,10$ \\
\hline Lecidella carpathica & $0 \cdot 2$ & $1,5,6,10$ \\
\hline Physconia americana & $0 \cdot 2$ & 6,10 \\
\hline P. californica & $0 \cdot 2$ & 6,10 \\
\hline P. enteroxantha & $0 \cdot 2$ & 1,6 \\
\hline Placopyrenium stanfordii & $0 \cdot 2$ & 1,3 \\
\hline Rhizocarpon viridiatrum & $0 \cdot 3$ & $2,6,10$ \\
\hline Xanthoparmelia verruculifera & $0 \cdot 2$ & 6,10 \\
\hline \multicolumn{3}{|l|}{ Non-ultramafic } \\
\hline \multicolumn{3}{|l|}{ Genus } \\
\hline Physconia & $-0 \cdot 4$ & $\mathbf{1}(1), 4(3), 5(3), \mathbf{1 0}(1)$ \\
\hline Rinodina & -0.6 & $4(3), 5(1), 6(1), 7(3)$ \\
\hline \multicolumn{3}{|l|}{ Species } \\
\hline Acarospora americana & $-0 \cdot 2$ & $\mathbf{1}, 5,7,8,9, \mathbf{1 0}$ \\
\hline Rinodina obnascens & $-0 \cdot 2$ & 4,5 \\
\hline Sarcogyne arenosa & $-0 \cdot 2$ & 5,7 \\
\hline Trapelia obtegens & $-0 \cdot 2$ & 7,8 \\
\hline Verrucaria fuscoatroides & $-0 \cdot 2$ & 1,9 \\
\hline
\end{tabular}

in relation to rock mineralogy, elemental chemistry, and geochemical processes is critical to advance understudied areas of lichenology, particularly physiological ecology and evolution. Thus, despite the obvious relationship between substratum and lichens, there still remains a critical need for the systematic description and characterization of lichen communities in relation to specific lithologies and chemical environments.

Our study is one of only a few to relate lichen occurrence to geochemistry of indi- vidual rocks (Boyle et al. 1987) (Table 2). Only four species were shared in common between all three substrata, suggesting substantial differences in lichen community composition between ultramafic and nonultramafic rocks at both the species and generic levels (Tables 5 \& 6). Brodo (1973) lists texture, water relations, and chemistry as the main factors that determine the composition of a lichen biota of a substratum. However, determining whether the differences we observed in lichen assemblages 
TABLE 6. Species richness values for sites (see Table 3), and per square decametre (see Table 1) compared with total lichen samples collected. Site scores are from a perMANOVA model of lichen species assembly between ultramafic $(\mathrm{n}=55)$ and non-ultramafic $(\mathrm{n}=5)$ sites

\begin{tabular}{llcccc}
\hline Site & \multicolumn{1}{c}{$\begin{array}{c}\text { Substratum } \\
\text { class }\end{array}$} & $\begin{array}{c}\text { Total number } \\
\text { of samples } \\
\text { collected }\end{array}$ & $\begin{array}{c}\text { Species } \\
\text { Richness/Site } \\
\text { Area }\end{array}$ & $\begin{array}{c}\text { Species } \\
\text { Richness/10 m }{ }^{2}\end{array}$ & $\begin{array}{c}\text { Site Score } \\
\text { (perMANOVA) }\end{array}$ \\
\hline 1 & ultramafic & 45 & 41 & $6 \cdot 94$ & $-0 \cdot 137$ \\
2 & ultramafic & 4 & 6 & $1 \cdot 76$ & $-0 \cdot 113$ \\
3 & ultramafic & 3 & 3 & $3 \cdot 33$ & $-0 \cdot 154$ \\
4 & non-ultramafic & 7 & 7 & $0 \cdot 19$ & $0 \cdot 077$ \\
5 & non-ultramafic & 37 & 29 & $0 \cdot 72$ & $0 \cdot 080$ \\
6 & ultramafic & 28 & 24 & $2 \cdot 70$ & $-0 \cdot 117$ \\
7 & non-ultramafic & 10 & 4 & $0 \cdot 04$ & $0 \cdot 156$ \\
8 & non-ultramafic & 2 & 17 & $0 \cdot 07$ & $0 \cdot 148$ \\
9 & non-ultramafic & 27 & 37 & $0 \cdot 03$ & $0 \cdot 086$ \\
10 & ultramafic & 38 & & $8 \cdot 80$ & $-0 \cdot 115$ \\
\hline
\end{tabular}

were due to the elemental content of the rocks, their physical properties, or age of the exposed rock surfaces was beyond the scope of this study. Generally, ultramafic rock outcrops are thought to support lichen taxa characteristic of exposed, sunny areas, those that have wide ecological amplitude, or taxa that colonize stressful habitats with reduced competition (Purvis 1996; Favero-Longo et al. 2004; Harris et al. 2007). Additionally, the lichen biota of ultramafic substrata appears to consist of a mixture of species having a high affinity for Si-rich and Ca-rich rocks (Purvis 1996; Favero-Longo et al. 2004). The lichen biota of the New Idria serpentinite mass is generally consistent with these characteristics, and confirms the higher species diversity on ultramafic rocks than on other rock types already reported from other sites (Gilbert \& James 1987; Sirois et al. 1988; Piervittori et al. 2004; Harris et al. 2007; Favero-Longo \& Piervittori 2009), although this may be due to the physical properties of the rock and/or the history of disturbance (see below). Wirth (1972) characterizes the ultramafic lichen communities of Central Europe by the absence or scarcity of lichens typical of Si-rich rocks [e.g. Rhizocarpon geographicum, Acarospora fuscata, Lasallia pustulata, Lecanora rupicola, Xanthoparmelia conspersa (as Parmelia conspersa)], the absence of species typical of base-rich rocks, and the occurrence of species at the northernmost limit of their ranges. Interestingly, the only two species found during the present study that were reported as scarce on ultramafic rocks by Wirth (viz. Rhizocarpon geographicum and Lecanora rupicola) occurred only on ultramafic rocks, which supports the hypothesis that the physical properties of the rock may be more important in determining lichen assemblages than their mineralization.

Bates (1978) suggested that lichen communities on ultramafic rocks were affected by the low availability of essential macronutrients such as $\mathrm{N}, \mathrm{P}, \mathrm{K}, \mathrm{S}$, and $\mathrm{C}$, and/or high concentrations of $\mathrm{Mg}$. Combined $\mathrm{Ca}$ deficiency and $\mathrm{Mg}$ toxicity results in the extreme adverse substratum condition of $\mathrm{Ca}: \mathrm{Mg}$ molar ratio $\ll 1$ (Brooks 1987). Ca is a plant-essential macronutrient and required in much higher concentrations than $\mathrm{Mg}$ (Marschner 2002). The two cations compete with each other for uptake at the root, and vascular plants with Type I cell walls (dicotyledon and most monocotyledon plants) contain cell walls that are highly dependent upon Ca-bridged pectins to maintain cell wall integrity (Marschner 2002; O'Dell \& Rajakaruna 2011). Unlike most vascular plants, the cell walls of fungi lack pectin (Kirk et al. 2011) and therefore fungi probably do not depend on an adequate supply of $\mathrm{Ca}$ to maintain cell wall integrity. It is thus unlikely that the chemistry of ultramafic sub- 
strata affects the fungal component of lichens in the same way that it affects vascular plants. It is possible, however, that the green algal (Chlorophyta; cell wall type similar to Type I) symbiont of lichens may be adversely affected by ultramafic substrata in the same manner as vascular plants since Ca-deficiency symptoms have been demonstrated for the non-lichenized, green algae Scenedesmus intermedius Chod. in a laboratory setting (Adam \& Issa 2000).

Heavy metal toxicity is another possible influence of ultramafic substrata on lichen species diversity and cover. Ultramafic substrata contain elevated concentrations of $\mathrm{Ni}$, $\mathrm{Cr}$, and other heavy metals (Brooks 1987). Many lichen species secrete oxalic acid, which weathers ultramafic rock and dissolves metals bound in minerals, thus increasing their bioavailability (e.g. Wilson et al. 1981). It is possible that the heavy metals contained in ultramafic rocks could potentially be toxic to lichens. Likewise, lichens growing on ultramafic rocks may be physiologically adapted to tolerate high heavy metal concentrations, such as that demonstrated on $\mathrm{Fe}$ and $\mathrm{Cu}$ smelter slag (Lange \& Ziegler 1963). Substitution of heavy metals by magnesium in one chemical compound in Tephromela atra (Huds.) Hafellner (as Lecanora atra) on serpentinites was reported by Wilson et al. (1981) as a possible method of avoiding the effects of toxic elements. More generally, it is evident that oxalates of a range of elements can form directly as a result of precipitation by reaction with oxalic acid during lichen growth (Purvis 1984). Ultramafic rocks (nephrite, partially serpentinized peridotite, serpentinite) analyzed from lichen collection sites of the New Idria serpentinite mass have 37 times as much $\mathrm{Ni}$ (2084 ppm vs. $109 \mathrm{ppm})$ and 16 times as much $\mathrm{Cr}$ (1810 ppm vs. 56 $\mathrm{ppm}$ ) than the non-ultramafic rocks analyzed (silica-carbonate; shale and sandstone). Which element or combination of elements may be critical in limiting lichen colonization remains elusive without element- and species-specific studies exploring the tolerance of various lichens to the significant elemental differences we observed among the rocks studied (Table 2).
The fact that the patterns of diversity and cover of lichens on ultramafic as compared to non-ultramafic rocks can be widely variable (Favero-Longo et al. 2004, and references therein), suggests climate, elevation, history of land use, and other biotic and abiotic factors may complicate the substratumlevel influence on lichens. The diverse lichen community we documented on ultramafic rocks of the New Idria serpentinite mass could be the result of the physical properties of the substratum (texture of the rocks) rather than due solely to their mineralogy. Ultramafic rocks of the sites from which we collected were typically hard with lamellar, granular, or vacuolar porous surface texture. In contrast, the non-ultramafic rocks were typically softer with vacuolar surface texture in the case of the silica-carbonate rock, and granular surface texture in the case of the shale and sandstone rock. Overall, the nonultramafic rocks tended to have more friable surfaces that may be too unstable to permit the establishment of a diverse lichen biota. Similarly, hard-weathering serpentinites of alpine habitats were shown to host higher lichen diversity and cover than soft-weathering rocks such as calc-schists (Favero-Longo \& Piervittori 2009).

An alternative explanation may be the difference in rock surface ages between the ultramafic and non-ultramafic sites. Most, or portions, of the non-ultramafic sites have been extensively disturbed by mining within the past 62 years, creating fresh rock surfaces, whereas virtually none of the ultramafic sites have been disturbed within the same time period (and probably for much longer).

Our study is the second account published to date of lichens collected from ultramafic rocks of the biodiverse California Floristic Province (Myers et al. 1999). Sigal (1989) provided the earlier account of ultramaficassociated lichens in central California, excluding the New Idria serpentinite mass, reporting 76 taxa from five sites. Although taxonomic concepts have changed since Sigal's study, and in some cases it is not possible to ascertain which species was actually recorded in her study, we report approximately the same 
number of species (83), only 15 of which were also reported in the earlier study (Table 3 ). The reasons for this are unclear, but possible factors are that Sigal also included species reported from soil, and that three out of the five study sites were significantly further north in the state than the New Idria serpentinite mass. To date, no endemic lichens have been reported from any of the ultramafic sites in California (or North America), although further taxonomic and phylogenetic studies may reveal distinct ecotypes or species. It is intriguing that despite the well-known phenomenon of ultramafic (or substratum-level) endemism in vascular plants (Anacker et al. 2011), species-level endemism is not a common phenomenon among cryptogams, including lichens (Sigal 1975) and bryophytes (Shaw et al. 1987; Lepp 2001; Briscoe et al. 2009). It is tempting to hypothesize that species- and community-level processes are more strongly influenced by other abiotic or biotic factors (e.g. microclimate, rock texture) than rock or soil mineralogy and, perhaps, the processes of speciation in cryptogams are less affected by isolation due to substratum chemistry (and other edaphic factors), known to be immensely important in generating diversity among vascular plants (Kruckeberg 1986; Rajakaruna 2004; Kay et al. 2011).

Of the 83 taxa (including four lichenicolous fungi) that we collected from ultramafic substrata, only 20 (Table 3) were included in the list of c. 250 lichen taxa reported by more than one ultramafic survey given by FaveroLongo et al. (2004). This is largely explained by the lack of studies devoted to lichens on ultramafic substrata in western North America. Interestingly, the two species from ultramafic substrata new to California were also two of those already reported from this substratum elsewhere by Favero-Longo et al. (2004): Buellia aethalea is a frequent species of hard, silica-rich rocks in Europe, and $B$. ocellata is a frequent species on ultramafic substrata (Favero-Longo et al. 2004) and was reported as new to New England from partially serpentinized peridotite by Harris et al. (2007). The two species reported from silica-carbonate are rare species, apparently restricted to calcareous sandstone in western USA, although $R$. saurinum has recently been reported from soft, aeolian sandstone in eastern Iran (Moniri et al. 2010). The two species reported from shale and sandstone are widespread but inconspicuous species that have probably been overlooked by previous workers.

Ultramafic substrata and other edaphically unusual habitats are undergoing drastic changes due to ever-expanding development, deforestation, mining, exotic species invasions, and atmospheric deposition of pollutants such as heavy metals or previously limiting nutrients such as nitrogen (Williamson \& Balkwill 2006; Rajakaruna \& Boyd 2008; Harrison \& Rajakaruna 2011). Such changes can have a drastic impact on the biota of these unique habitats. Floristic surveys in support of conservation efforts should be encouraged to document the wealth of biological diversity being frequently lost from such sites worldwide. These sites, perhaps one of the last remaining under-studied frontiers of genetic diversity, should be better explored to generate data for effective conservation planning.

We thank Othmar Breuss (Vienna, Austria), Theodore Esslinger (Fargo, North Dakota, USA), Anna Guttová (Bratislava, Slovakia), and John Sheard (Saskatoon, Saskatchewan, Canada) for assistance with identifying our collections; Jill Lee for assistance with preparing tables; Laureen Wagoner for providing details on the $\mathrm{XRF}$ analysis; Bruce McCune and an anonymous reviewer for providing useful comments for an earlier version of the manuscript; Maine Space Grant Consortium, College of the Atlantic, and San José State University for providing generous funding for the project.

\section{REFERENCES}

Adam, M. S. \& Issa, A. A. (2000) Effect of manganese and calcium deficiency on the growth and oxygen exchange of Scenedesmus intermedius cultured for successive generations. Folia Microbiologica 45: 353358.

Alexander, E. B., Coleman, R. G., Keeler-Wolf, T. \& Harrison, S. P. (2007) Serpentine Geoecology of Western North America. New York: Oxford University Press.

Anacker, B. L., Whittall, J. B., Goldberg, E. B. \& Harrison, S. P. (2011) Origins and consequences of serpentine endemism in the California flora. Evolution 65: 365-376. 
Anderson, M. J. (2001) A new method for non-parametric multivariate analysis of variance. Austral Ecology 26: 32-46.

Bates, J. W. (1978) The influence of metal availability on the bryophyte and macrolichen vegetation of four rock types on Skye and Rhum. Fournal of Ecology 66: $457-482$.

Boyle, A. P., McCarthy, P. M. \& Stewart, D. (1987) Geochemical control of saxicolous lichen communities on the Creggaun Gabbro, Letterfrack, Co. Galway, western Ireland. Lichenologist 19: 307-317.

Briscoe, L. R. E., Harris, T. B., Dannenberg, E., Broussard, W., Olday, F. C. \& Rajakaruna, N. (2009) Bryophytes of adjacent serpentine and granite outcrops on the Deer Isles, Maine, USA. Rhodora 111: $1-20$.

Brodo, I. M. (1973) Substrate ecology. In The Lichens (V. Ahmadjian \& M. E. Hale, eds): 401-441. New York: Academic Press.

Brooks, R. R. (1987) Serpentine and its Vegetation: $A$ Multidisciplinary Approach. Portland, Oregon: Dioscorides Press.

Coleman, R. G. \& Jove, C. (1992) Geological origin of serpentinites. In The Vegetation of Ultramafic (Serpentine) Soils (A. J. M. Baker, J. Proctor \& R. D. Reeves, eds): 1-17. Andover, Hampshire: Intercept.

Esslinger, T. L. (2011) A cumulative checklist for the lichen-forming, lichenicolous and allied fungi of the continental United States and Canada. North Dakota State University: http://www.ndsu.edu/ pubweb/ esslinge/chcklst/chcklst7.htm [First Posted 1 December 1997, Most Recent Version (\#17) 16 May 2011], Fargo, North Dakota.

Faith, D. P., Minchin, P. R. \& Belbin, L. (1987) Compositional dissimilarity as a robust measure of ecological distance. Vegetatio 69: 57-68.

Favero-Longo, S. E. \& Piervittori, R. (2009) Measuring the biodiversity of saxicolous lichens above timberline with reference to environmental factors: the case-study of a Natura 2000 site of western Alps. Phytocoenologia 39: 51-78.

Favero-Longo, S. E., Isocrono, D. \& Piervittori, R. (2004) Lichens and ultramafic rocks: a review. $\mathrm{Li}$ chenologist 36: 391-404.

Favero-Longo, S. E., Castelli, D., Salvadori, O., Belluso, E. \& Piervittori, R. (2005) Pedogenetic action of the lichens Lecidea atrobrunnea, Rhizocarpon geographicum gr. and Sporastatia testudinea on serpentinized ultramafic rocks in an alpine environment. International Biodeterioration and Biodegradation 56: 17-27.

García, L. V. (2003) Controlling the false discovery rate in ecological research. Trends in Ecology and Evolution 18: 553-554.

Garty, J. \& Galun, M. (1974) Selectivity in lichensubstrate relationships. Flora 163: 530-534.

Gilbert, C. A. (1984) Prospectors, capitalists and bandits: the history of the New Idria Quicksilver Mine, 18541972. M.S. thesis, San Jose State University.

Gilbert, O. L. \& James, P. W. (1987) Field meeting on the Lizard Peninsula, Cornwall. Lichenologist 19: 319-334.
Hafellner, J. (1991) Die Flechtenflora eines hochgelegenen Serpentinitstockes in den Ostalpen (Österreich, Steiermark). Mitteilungen der Naturwissenschaftlichen Vereines für Steiermark 121: 95-106.

Harris, T. B., Olday, F. C. \& Rajakaruna, N. (2007) Lichens of Pine Hill, a peridotite outcrop in Eastern North America. Rhodora 109: 430-447.

Harrison, S. P. \& Rajakaruna, N. (eds). (2011) Serpentine: Evolution and Ecology in a Model System. Berkeley, California: University of California Press.

Hauck, M., Huneck, S., Elix, J. A. \& Paul, A. (2007) Does secondary chemistry enable lichens to grow on iron-rich substrates? Flora 202: 471-478.

Hausdorf, B. \& Hennig, C. (2005) The influence of recent geography, palaeogeography and climate on the composition of the fauna of the central Aegean Islands. Biological Fournal of the Linnaean Society 84: 785-795.

Hegetschweiler, C. \& Stizenberger [E.] (1887) Mittheilung über lichenen auf ungewöhnlichem substrate. Flora 70: 430-431.

Hothorn, T., Hornik, K., van de Wiel, M. A. \& Zeileis, A. (2008) Implementing a class of permutation tests: the coin package. Fournal of Statistical Software 28: $1-23$.

Index Fungorum Partnership. (2010+) Index Fugorum. A community resource. CABI, CBS, and Landcare Research, custodians. CABI, Wallingford, Oxfordshire, UK; CBS KNAW Fungal Biodiversity Centre, Utrecht, The Netherlands; and Manaaki Whenua Landcare Research, Lincoln, New Zealand. Website (http://www.indexfungorum.org).

Kay, K. M., Ward, K. L., Watt, L. R. \& Schemske, D. W. (2011) Plant speciation. In Serpentine: Evolution and Ecology in a Model System (S. P. Harrison \& N. Rajakaruna, eds): 71-96. Berkeley, California: University of California Press.

Kirk, P. M., Cannon, P. F., Minter, D. W. \& Stalpers, J. A. (eds) (2011) Dictionary of the Fungi 10th edn. Egham, UK: CAB International.

Kossowska, M. (2001) Epilithic lichens on serpentinite rocks in Poland. Polish Botanical fournal 46: 191197.

Kruckeberg, A. R. (1986) An essay: the stimulus of unusual geologies for plant speciation. Systematic Botany 11: 455-463.

Lange, O. L. \& Ziegler, H. (1963) Der Schwermetallgehalt von Flechten aus dem Acarosporetum sinopicae auf Erzschlackenhalden des Harzes. I. Eisen und Kupfer. Mitteil. der Florist-soziologischen Arbeitsgemeinschaft, N. F. 10: 156-183.

Lazarus, B. E., Richards J. H., Claassen, V. P., O’Dell, R. E. \& Ferrell, M. A. (2011) Species specific plant-soil interactions influence plant distribution on serpentine soils. Plant and Soil 342: 327-344.

Legendre, P. (2007) One-way ANOVA with permutation test. Website (http://www.bio.umontreal.ca/ Casgrain/prog/labo/fonctions_r/anova.1way.R.zip)

Lendemer, J. C., Knudsen, K. \& Coppins, B. J. (2009) Further notes on the genus Ramonia in California: the first modern record of $R$. ablephora and the 
description of $R$. extensa sp. nov. Opuscula Philolichenum 7: 191-194.

Lepp, N. W. (2001) Bryophytes and pteridophytes. In Metals in the Environment-Analysis by Biodiversity (M. N. V. Prasad, ed): 199-204. New York: Marcel Dekker.

Marschner, H. (2002) Mineral Nutrition of Higher Plants. San Diego: Academic Press.

McArdle, B. H. \& Anderson, I. C. (2001) Fitting multivariate models to community data: a comment on distance-based redundancy analysis. Ecology 82: 290-297.

Moniri, M. H., Kamyabi, S. \& Fryday, A. M. (2010) Rhizocarpon saurinum new to Asia, and other reports of Rhizocarpon species from Razavi Khorasan Province, Iran. Mycologia Balcanica 6: 89-92.

Myers, N., Mittermeier, R. A., Mittermeier, C. G., Da Fonseca, G. A. B. \& Kent, J. (1999) Biodiversity hotspots for conservation priorities. Nature 403: 853-858.

O'Dell, R. E. \& Rajakaruna, N. (2011) Intraspecific variation, adaptation, and evolution. In Serpentine: Evolution and Ecology in a Model System (S. P. Harrison \& N. Rajakaruna, eds): 97-137. Berkeley, California: University of California Press.

Oksanen, J., Kindt, R., Legendre, P., O’Hara, B., Simpson, G. L., Solymos, P., Stevens, M. H. H. \& Wagner, H. (2011) R package version 1.15-3. Community Ecology Package, vegan. URL: http:// vegan.r-forge.r-project.org/

Paukov, A. G. (2009) The lichen flora of serpentine outcrops in the Middle Urals of Russia. Northeastern Naturalist 16: 341-350.

Piervittori, R., Isocrono, D., Favero-Longo, S. E. \& De Nicolò, A. (2004) Indagini floristiche ed ecologichesui licheni degli ambienti ofiolitici del Parco Naturale del Mont Avic: influenza della naturageologica del substrato sulle comunità licheniche rupicole e terricole. Revue Valdôtain Histoire Naturelle 58: 51-64.

Purvis, O. W. (1984) The occurrence of copper oxalate in lichens growing on copper sulphide-bearing rocks in Scandinavia. Lichenologist 16: 197-204.

Purvis, O. W. (1996) Interactions of lichens with metals. Science Progress 79: 283-309.

Purvis, O. W. \& Halls, C. (1996) A review of lichens in metal-enriched environments. Lichenologist 28: 571601.

Purvis, O. W. \& Pawlik-Skowrońska, B. (2008) Lichens and metals. In Stress in Yeasts and Filamentous Fungi. British Mycological Society Symposium Series (S. Avery, M. Stratford \& P. van West, eds): 175-200. Amsterdam: Elsevier \& Academic Press.

R Development Core Team. (2011) R: A Language and Environment for Statistical Computing. R Foundation for Statistical Computing, Vienna, Austria. ISBN 3900051-07-0, URL http://www.R-project.org/.
Rajakaruna, N. (2004) The edaphic factor in the origin of species. International Geology Review 46: 471478.

Rajakaruna, N. \& Boyd, R. S. (2008) The edaphic factor. In The Encyclopedia of Ecology. Vol. 2 (S. E. Jorgensen \& B. Fath, eds): 1201-1207. Oxford: Elsevier.

Rajakaruna, N., Harris, T. B. \& Alexander, E. B. (2009) Serpentine geoecology of eastern North America: a review. Rhodora 111: 21-108.

Rajakaruna, N., Harris, T. B., Clayden, S., Dibble, A. \& Olday, F. S. (2011) Lichens of Callahan Mine, a copper and zinc-enriched Superfund site in Brooksville, Maine, U.S.A. Rhodora 113: 1-31.

Richardson, D. H. S. (1995) Metal uptake in lichens. Symbiosis 18: 119-127.

Rune, O. (1954) Notes on the flora of the Gaspé Peninsula. Svensk Botanisk Tidskrift 48: 117-138.

Ryan, B. D. (1988) Marine and maritime lichens on serpentine rock on Fidalgo Island, Washington. Bryologist 91: 186-190.

Shaw, A. J., Antonovics, J. \& Anderson, L. E. (1987) Inter- and intraspecific variation of mosses in tolerance to copper and zinc. Evolution 41: 1312-1325.

Shimizu, A. (2004) Community structure of lichens in the volcanic highlands of Mt. Tokachi, Hokkaido, Japan. Bryologist 107: 141-151.

Sigal, L. L. (1975) Lichens and mosses of California serpentine. M.A. thesis, San Fransisco State University, California.

Sigal, L. L. (1989) The lichens of serpentine rocks and soils in California. Mycotaxon 34: 221-238.

Sirois, L., Lutzoni, F. \& Grandtner, M. M. (1988) Les lichens sur serpentine et amphibolite du plateau du mont Albert, Gaspésie, Québec. Canadian fournal of Botany 66: 851-862.

Van Baalen, M. R. (1995) The New Idria serpentinite. Ph.D. thesis, Harvard University.

von Brackel, W. (2007) Zur Flechtenflora der Serpentinitfelsen in Nordostbayern. Hoppea, Denkschriften der Regensburgischen Botanischen Gesellschaft 68: 253268.

Werner, R. G. (1956) Etudes ecologiques sur les lichens des terrains schisteux maritimes. Bulletin de la Société des Sciences de Nancy, n. ser. 15: 137-152.

Williamson, S. D. \& Balkwill, K. (2006) Factors determining levels of threat to serpentine endemics. South African fournal of Botany 72: 619-626.

Wilson, M. J. (1995) Interactions between lichens and rocks: a review. Cryptogamic Botany 5: 299-305.

Wilson, M. J., Jones, D. \& McHardy, W. J. (1981) The weathering of serpentinite by Lecanora atra. Lichenologist 13: 167-176.

Wirth, V. (1972) Die Silikatflechten-Gemeinschaften in Ausseralpinen Zentraleuropa. Dissertationes Botanicae 17: 1-305. 